\title{
IMMERSIONS AND EMBEDDINGS IN DOMAINS OF HOLOMORPHY
}

\author{
AVNER DOR
}

\begin{abstract}
Let $D_{1}$ be a bounded smooth strongly pseudoconvex domain in $\mathbb{C}^{N}$ and let $D_{2}$ be a domain of holomorphy in $\mathbb{C}^{M}(2 \leq N, 5 \leq M, 2 N \leq M)$. There exists then a proper holomorphic immersion from $D_{1}$ to $D_{2}$. Furthermore if $\operatorname{PI}\left(D_{1}, D_{2}\right)$ is the set of proper holomorphic immersions from $D_{1}$ to $D_{2}$ and $A\left(D_{1}, D_{2}\right)$ is the set of holomorphic maps from $D_{1}$ to $D_{2}$ that are continuous on the boundary, then the closure of $\operatorname{PI}\left(D_{1}, D_{2}\right)$ in the topology of uniform convergence on compacta contains $A\left(D_{1}, D_{2}\right)$. The approximating proper maps can be made tangent to any finite order of contact at a given point. The same result was obtained for proper holomorphic maps, in one codimension, when the target domain has a plurisubharmonic exhaustion function with no saddle critical points. This includes the case where the target domain is convex. Density in a weaker sense was derived in one codimension when the critical points are contained in a compact subset of the target domain. This occurs (for example) when the target domain is bounded weakly pseudoconvex with $C^{2}$-smooth boundary. If the target domain is strongly pseudoconvex then the approximating proper holomorphic maps can also be made continuous on the boundary.

A lesser degree of pseudoconvexity is required from the target domain when the codimension is larger than the minimal. A domain in $\mathbb{C}^{L}$ is called " $M$ dimensional-pseudoconvex" (where $L \geq M$ ) if it has a smooth exhaustion function $r$ such that every point $w$ in this domain has some $M$-dimensional complex affine subspace going through this point for which $r$, restricted to this subspace, is strictly plurisubharmonic in $w$. In the result mentioned above the assumption that the target domain is pseudoconvex in $\mathbb{C}^{M}(M \geq 2 N, 5)$ can be substituted for the assumption that the domain is " $M$-dimensionalpseudoconvex". Similarly, the assumption that the target domain $D_{2}$ is " $N+$ 1)-dimensional-pseudoconvex" and all the critical points of some appropriate exhaustion function are " $(N+1)$-dimensional-convex" (defined in a similar manner) yields that the closure of the set of proper holomorphic maps from $D_{1}$ to $D_{2}$ contains $A\left(D_{1}, D_{2}\right)$.

All the results are obtained with embeddings when the Euclidean dimensions are such that $\operatorname{dim}_{\mathbb{C}}\left(D_{2}\right) \geq 2 \operatorname{dim}_{\mathbb{C}}\left(D_{1}\right)+1$. Thus, in this case, when one of the assumptions mentioned above is fulfilled, then the closure of the set of embeddings from $D_{1}$ to $D_{2}$ contains $A\left(D_{1}, D_{2}\right)$.
\end{abstract}

\section{INTRODUCTION}

When $D \subset \mathbb{C}^{N}, \Omega \subset \mathbb{C}^{M}$ are domains, we denote by $A(D, \Omega)$ the set of continuous maps from $\bar{D}$ to $\Omega$ that are holomorphic in $D$. The set of proper

Received by the editors July $29,1993$.

1991 Mathematics Subject Classification. Primary 32H35. 
holomorphic maps from $D$ to $\Omega$ will be denoted by $\mathbf{P}(D, \Omega)$, and $\mathbf{P I}(D, \Omega)$ will be the set of proper holomorphic immersions from $D$ to $\Omega$. The set of injective maps in $\operatorname{PI}(D, \Omega)$ (which are the embeddings from $D$ to $\Omega$ ) will be denoted by $\mathbf{E}(D, \Omega)$.

Theorem 1. Take $N \geq 2$ and $M \geq \max \{2 N, 5\}$. Let $\Omega \subset \mathbb{C}^{M}$ be a domain of holomorphy, and let $D \subset \subset \mathbb{C}^{N}$ be a strongly pseudoconvex domain with $C^{\infty}$ boundary. There then exists a proper holomorphic immersion from $D$ to $\Omega$. Furthermore, the closure of $\mathbf{P I}(D, \Omega)$, in the topology of uniform convergence on compacta, contains $A(D, \Omega)$. When $M>2 N$ there exists an embedding from $D$ to $\Omega$ and the closure of $\mathbf{E}(D, \Omega)$, in the same topology, contains $A(D, \Omega)$. Moreover, if we take $f \in A(D, \Omega), K \subset D$ compact, $\varepsilon>0, P \in D, k \geq 0$, then:

(1) There exists $F \in \mathbf{P}(D, \Omega)$ such that $|F-f|<\varepsilon$ on $K$ and $D^{\alpha} F(P)=$ $D^{\alpha} f(P)$ for all $\alpha \in \mathbf{N}^{N},|\alpha| \leq k$ (in particular $F(P)=f(P)$ ).

(2) There exists $F \in \mathbf{P I}(D, \Omega)$ such that $|F-f|<\varepsilon$ on $K, F(P)=f(P)$ and $D^{\alpha} F(P)=D^{\alpha} f(P)$ for all $\alpha \in \mathbf{N}^{N}, 2 \leq|\alpha| \leq k$.

(3) In the case that $M \geq 2 N+1$, then $F$ in (1) and (2) can also be one to one. (When $\alpha=\left(\alpha_{1}, \ldots, \alpha_{N}\right) \in \mathbf{N}^{N}=\mathbf{N} \times \cdots \times \mathbf{N}$, the notation $|\alpha|$ stands for: $\alpha_{1}+\cdots+\alpha_{n}$.)

In both cases (when $M=2 N$ and $M>2 N) F$ can be (in addition) continuous on $\bar{\Omega}$ when $\Omega$ is bounded strongly pseudoconvex with $C^{2}$ boundary.

Let $\rho$ be a smooth plurisubharmonic exhaustion function of the target domain $\Omega$. The main part of our proof consists of an infinite inductive process of correcting $f$ so that $\rho \circ f$ will be larger on $b D$. A part of this paper, centered around Lemma 1, deals with pushing the image of $b D$ toward the boundary of $\Omega$, through parts of $\Omega$ that are away from critical points of $\rho$. For this, one codimension will suffice. The critical points of $\rho$ then prove to be a major obstacle. Very hard work and some additional codimension (as in Theorem 1) are needed to push the map through them. However, this is true only for critical points which are not strict local minima. Those that are strict local minima are harmless at any positive codimension. Thus we will obtain in one codimension a weaker density result when the saddle critical points (i.e. the critical points which are not strict local minima) are contained in a compact subset. This condition holds for smooth bounded weakly pseudoconvex domains.

Theorem 2. Let $N \geq 2, M \geq N+1$ and $D \subset \subset \mathbb{C}^{N}$ be as in Theorem 1 .

(I) Let $\Omega \subset \mathbb{C}^{M}$ be a pseudoconvex domain (without boundedness or smoothness assumptions) which has a $C^{2}$ strictly plurisubharmonic exhaustion function $\rho$, and let $a \in \mathbb{R}$, such that all the saddle critical points of $\rho$ are contained in the set $\{w \in \Omega: \rho(w) \leq a\}$. Then there exists a proper holomorphic map from $D$ to $\Omega$.

Furthermore, take $f \in A(D, \Omega)$, where $f(b D) \subset\{w \in \Omega: \rho(w)>a\}$; take also $K \subset D$ compact, $\varepsilon>0, P \in D, k \geq 0$. Then there exists $F \in \mathbf{P}(D, \Omega)$ such that $|F-f|<\varepsilon$ on $K$ and $D^{\alpha} F(P)=D^{\alpha} f(P)$ for all $\alpha \in \mathbf{N}^{N},|\alpha| \leq k$.

(II) Let $\Omega \subset \subset \mathbb{C}^{M}$ be a pseudoconvex domain with $C^{2}$ boundary. There exists then a neighborhood $G \subset \mathbb{C}^{M}$ of $b \Omega$ such that if $f \in A(D, \Omega), f(b D) \subset$ $G, K \subset D$ is compact, $\varepsilon>0, P \in D$, and $k \geq 0$, then there exists $F \in$ $\mathbf{P}(D, \Omega)$ such that $|F-f|<\varepsilon$ on $K$ and $D^{\alpha} F(P)=D^{\alpha} f(P)$ for all $\alpha \in$ 
$\mathbf{N}^{N},|\alpha| \leq k$. If $\Omega$ is a strongly pseudoconvex domain then $F$ can also be continuous on $\bar{\Omega}$.

Part (II) of Theorem 2 is a corollary of part (I). Another immediate, but important, corollary is the following.

Corollary 1. Let $N \geq 2, M \geq N+1$ and $D \subset \subset \mathbb{C}^{N}$ be as above. Let $\Omega \subset \mathbb{C}^{M}$ be a pseudoconvex domain (not necessarily bounded or smooth) which has a $C^{2}$ strictly plurisubharmonic exhaustion function $\rho$ with no saddle critical points. Then the closure of $\mathbf{P}(D, \Omega)$, in the topology of compact convergence, contains $A(D, \Omega)$. If we take $f \in A(D, \Omega), K \subset D$ compact, $\varepsilon>0, P \in D, k \geq 0$, then there exists $F \in \mathbf{P}(D, \Omega)$ such that $|F-f|<\varepsilon$ on $K$ and $D^{\alpha} F(P)=$ $D^{\alpha} f(P)$ for all $\alpha \in \mathbf{N}^{N},|\alpha| \leq k$.

It is natural to ask whether the pseudoconvexity assumptions, in these results, can be somewhat relaxed when the codimension is larger. Say, for example, that $M>M_{0}=\max \{2 N, 5\}$, and let $\Omega \subset \mathbb{C}^{M}$ be a domain with the assumption that for some $M_{0}$-dimensional affine subspace $V \subset \mathbb{C}^{M}$ the intersection $V \cap \Omega$ has a nonempty connected component which is a domain of holomorphy in $V$. Then (by Theorem 1) there exists a proper holomorphic immersion from $B^{N}$ to $\Omega$ which is contained in $V \cap \Omega$. While in this trivial situation we can say nothing about the density of $\operatorname{PI}(D, \Omega)$ in $A(D, \Omega)$; this line of thought will be developed in the next definition and theorem to a generalization of our results when the codimension is larger. Theorem 3 is derived from the proofs of Theorems 1, 2.

Definition. Let $1 \leq m \leq k$, let $G \subset \mathbb{C}^{k}$ be open and let $\rho: G \rightarrow \mathbb{R}$ be a $C^{2}$ function. The function $\rho$ is called " $m$-dimensional-plurisubharmonic" if for every $w \in G$ there exists an $m$-dimensional complex affine subspace $V$ where $w \in V$ and the restriction $\rho$ to $V \cap G$ is strictly plurisubharmonic in $w$. (Note that $V-w$ depends on $w$.)

A point $w \in G$ is called an " $m$-dimensional-convex point of of $\rho$ " if there exists an $m$-dimensional affine subspace $V$ where $w \in V$ and the restriction of $\rho$ to $V \cap G$ is strictly convex in $w$ (see Definition 1.5 below).

A domain $\Omega \subset \mathbb{C}^{k}$ is called " $m$-dimensional-pseudoconvex" if it has an exhaustion function which is $m$-dimensional-plurisubharmonic.

All our results hold (including the density and tangential parts) under the following weaker assumptions:

Theorem 3. (1) Theorem 1 fully holds under the assumption that $\Omega$ is $M_{0^{-}}$ dimensional-pseudoconvex, where $M_{0}=\max \{2 N, 5\}$. In particular the closure of $\mathbf{E}(D, \Omega)$ contains $A(D, \Omega)$ when the Euclidean dimensions yield $\operatorname{dim}(\Omega) \geq$ $2 \operatorname{dim}(D)+1$.

(2) Theorem 2 (I) fully holds when $\Omega$ has an $(N+1)$-dimensional-plurisubharmonic exhaustion function $\rho$ where (for some $a \in \mathbb{R}$ ) all the critical points of $\rho$ in the set $\{w \in \Omega: \rho(w)>a\}$ are $(N+1)$-dimensional-convex points of $\rho$.

(3) Corollary 1 fully holds when $\Omega$ has an $(N+1)$-dimensional-plurisubharmonic exhaustion function $\rho$ where all the critical points of $\rho$ are $(N+1)$ dimensional-convex points of $\rho$. In particular the closure of $\mathbf{P}(D, \Omega)$ contains $A(D, \Omega)$. 
(4) Theorem 2 (II) fully holds with the assumption that $\Omega$ is bounded $C^{2}$ smooth and $1 / d(w, b \Omega)$ is an $(N+1)$-dimensional-plurisubharmonic function near the boundary of $\Omega$.

Some applications and corollaries of Theorem 3 will be discussed later in the introduction. Theorems 1, 2, 3 and Corollary 1 are proved, to a large extent, together. This is described in the comments before and after Lemma 10.

Previously, a result similar to Theorem 1 was proven in one codimension $(M \geq N+1)$ in the case that $\Omega$ is a general convex domain (see [D3]). Corollary 1 generalizes the main part of that result. In [D2] a continuous proper holomorphic map into an arbitrary bounded $C^{2}$-smooth domain was constructed in one codimension. The map in [D2] was confined to a small neighborhood of a "convex corner" in the boundary of the target domain. By Theorem 2 above, in the weakly pseudoconvex case it is enough to assume that the map that we start with takes the boundary of $D$ close enough to the boundary of $\Omega$. In our main result, which is Theorem 1, and also in Corollary 1 and in Theorem 3 (parts (1) and (3)), the starting map, $f$, rests in complete freedom in the target domain. Here, we might have to get through critical points to push the map towards the boundary. When looking at all the constructions of proper holomorphic maps, one sees that prescribing the way the map sits inside the target domain is one of the most interesting and difficult problems in this field.

By a different type of proof Forstnerič and Globevnik showed in [FG, Theorem 2] the existence of a proper holomorphic map from $\Delta$ to a bounded $C^{2}$-smooth pseudoconvex domain in $\mathbb{C}^{M}, M \geq 2$. The map has a prescribed value and derivatives (up to a proportion) in the origin. Example 5 in their paper shows that the density result of Theorem 1 above does not hold for arbitrary bounded smooth domains, although, by [D2], such domains contain proper images of lower dimensional balls.

It would be interesting to now find necessary conditions under which the proper maps from $B^{N}, N \geq 1$, to a domain $\Omega \subset \mathbb{C}^{M}$ are dense in $A\left(B^{N}, \Omega\right)$. The main question is whether there are bounded target domains with a smooth and connected boundary which are not covered by Theorem 3 .

In [D5] it was verified that in the above-mentioned construction (in [D2]) of a proper holomorphic map into a bounded smooth domain, a smoothness assumption of the target domain is, in fact, necessary. An example is given of a bounded domain $\Omega \subset \mathbb{C}^{m}$ (for any $m \geq 2$ ) such that there is no proper holomorphic map from $\Delta$ to $\Omega$.

It is mentioned in [D2] that if $\Omega \subset \mathbb{C}^{M}$ is an arbitrary unbounded domain with $C^{2}$ boundary that has one point of strong pseudoconvexity on the boundary, then there exists a proper holomorphic map from $B^{N}$ to $\Omega$, where $M \geq N+1$. Therefore, by Theorem 2 , if we assume that $\Omega$ is an unbounded $C^{2}$-smooth weakly pseudoconvex domain, there would be a rather narrow situation in which the existence of one codimensional proper holomorphic map from the ball into such this domain is not verified. That is:

(*) When all points on the boundary of $\Omega$ are weakly pseudoconvex and all plurisubharmonic exhaustion functions of $\Omega$ have saddle critical points clustering on the boundary of $\Omega$.

The domains that satisfy $(*)$ are then the only $C^{2}$-smooth unbounded weakly pseudoconvex domains that are not known to contain proper images of a ball in 
one codimension. Note that there are such domains, for example, the domain:

$$
\left(\mathbb{C} \backslash \bigcup_{a, b \in \mathbb{Z}}(a+i b+(1 / 4) \bar{\Delta})\right) \times \mathbb{C}^{M-1} .
$$

While results proved in [D1, D2, D3, D4] are quoted and applied here, the methods of these papers are not sufficient for the pseudoconvex case and new types of correction functions are needed. At the end of this introduction we will give a short and simplified account of the role of these functions, which may be helpful in understanding the motivation of the technical and difficult proofs of Lemmas $1,2$.

In Theorems 1, 2, 3 and Corollary 1 the approximating map is tangent to the given map, in a point that we choose, to any order that we wish. We get from it the following corollary:

Corollary 2. Let $2 \leq N, M \geq \max \{2 N, 5\}, D \subset \subset \mathbb{C}^{N}$ as in Theorem 1, and let $\Omega \subset \mathbb{C}^{M}$ be a domain of holomorphy.

Take $P \in D, Q \in \Omega, k \geq 0$, and let $v_{\alpha}$ (where $\alpha \in \mathbf{N}^{N},|\alpha| \leq k$ ) be arbitrary given vectors in $\mathbb{C}^{M}$. There exist then $F \in \mathbf{P}(D, \Omega)$ and $t>0$, such that $F(P)=Q$ and $D^{\alpha} F(P)=t v_{\alpha}$ for all $\alpha \in \mathbf{N}^{N}$ where $1 \leq|\alpha| \leq k$. In the case that $M \geq 2 N+1, F$ can be 1-1.

In view of Theorem 3, it is enough to assume here that $\Omega$ is $M_{0}$-dimensionalpseudoconvex, where $M_{0}=\max \{2 N, 5\}$. The corollary also holds when $\Omega$ has an $(N+1)$-dimensional-plurisubharmonic exhaustion function $\rho$ where all the critical points of $\rho$ are $(N+1)$-dimensional-convex points of $\rho$. Similarly, (by Corollary 1) we can assume in Corollary 2 that $\Omega$ has a $C^{2}$ strictly plurisubharmonic exhaustion function $\rho$ with no saddle critical points. A similar corollary, in one codimension, can be derived from Theorem 2. Corollary 2 can be viewed as a multi-dimensional version of the above mentioned tangency property in the paper [FG].

In [D3] there is a construction of proper maps into convex domains from which a "thin" closed set is omitted. The results there can be generalized to the pseudoconvex case.

Definition. A subset $S$ of $\mathbb{C}^{M}$ will be called a " $k$-dimensional real subcurve" $(k \geq 0)$, if $S$ is a subset of a $C^{1}$ image of an open subset of $\mathbb{R}^{k}$.

Corollary 3. Let $k=2(M-N)-1$. Theorems 1, 2, 3 and Corollaries 1, 2 (with all their parts) hold when $\Omega$ is replaced by $\Omega^{\prime}=\Omega \backslash E$, where $E \subset \Omega$ is relatively closed and $E=\left(\bigcup_{1 \leq n<\infty} S_{n}\right) \cap \Omega$ where $S_{n} \subset \mathbb{C}^{M}(1 \leq n<\infty)$ are $k$-dimensional real subcurves.

The proof is an adaptation of the proof of Theorem 3 of [D3] to the proofs of Theorems 1, 2, 3 and Corollary 1 here. To this we add an element from the proof of Proposition 1 to maintain the tangency property $\left(D^{\alpha} F(P)=D^{\alpha} f(P)\right.$ for all $|\alpha| \leq k)$. The details are straightforward applications and we will not go through them.

By combining techniques from [D4] into this paper we can generalize the lifting result of [D4]. In the next corollary the ball $B^{N-1}$ is identified with $B^{N-1} \times\{0\} \subset B^{N}$. 
Corollary 4. Let $2 \leq N, M \geq \max \{2 N, 5\}$, and let $\Omega \subset \subset \mathbb{C}^{M}$ be a $C^{2}$-smooth strongly pseudoconvex domain. Then every map in $\mathbf{P}\left(B^{N-1}, \Omega\right)$ is a restriction to $B^{N-1}$ of a map in $\mathbf{P}\left(B^{N}, \Omega\right)$. When $\Omega$ is strongly convex it is enough to assume $M \geq N+1$.

Examples of applications of Theorem 3. In the following discussion $N \geq 2$, and $D \subset \subset \mathbb{C}^{N}$ is an arbitrary $C^{\infty}$-smooth strongly pseudoconvex domain.

(1) Let $\Omega=\left\{z \in \mathbb{C}^{4}:\left|z_{1}\right|^{2}+\left|z_{2}\right|^{2}+\left|z_{3}\right|^{2}-\exp \left(\left|z_{4}\right|^{2}\right)<0\right\}$; then $\mathbf{P}\left(B^{2}, \Omega\right)$ is dense in $A\left(B^{2}, \Omega\right)$.

(2) More generally: Let $M \geq N+2$, and let $r$ be the exhaustion function of a domain $\Omega \subset \mathbb{C}^{M}$, where: $r\left(w_{1}, \ldots, w_{M}\right)=r_{1}\left(w_{1}, \ldots, w_{N+1}\right)+$ $r_{2}\left(w_{N+2}, \ldots, w_{M}\right), r_{1}$ is a $C^{2}$ strictly plurisubharmonic function with no saddle critical points and $r_{2}$ is an arbitrary $C^{2}$ function. Then $\mathbf{P}(D, \Omega)$ is dense in $A(D, \Omega)$.

(3) Let $M_{0}=\max \{2 N, 5\}, M>M_{0}$, and let $r$ be the exhaustion function of a domain $\Omega \subset \mathbb{C}^{M}$, where: $r\left(w_{1}, \ldots, w_{M}\right)=r_{1}\left(w_{1}, \ldots, w_{M_{0}}\right)+$ $r_{2}\left(w_{M_{0}+1}, \ldots, w_{M}\right)+r_{3}\left(w_{1}, \ldots, w_{M}\right)$, such that: $r_{1}$ is a $C^{2}$ strictly plurisubharmonic function, $r_{2}$ is an arbitrary $C^{2}$ function and $r_{3}$ is any given $C^{2}$ function with a smaller complex hessian than $r_{1}$, in the variables $w_{1}, w_{2}, \ldots, w_{M_{0}}$. Then $\mathbf{E}(D, \Omega)$ is dense in $A(D, \Omega)$.

(4) Let $M_{0}=\max \{2 N, 5\}, M>M_{0}$, and let $\Omega^{\prime} \subset \mathbb{C}^{N}$ be a domain of holomorphy. Let $\Omega \subset \mathbb{C}^{M}$ be a domain such that there exists a diffeohomeomorphism $f: \Omega \rightarrow \Omega^{\prime}$ where for every $w \in \Omega$ there exists an $M_{0}$-dimensional affine subspace $V$, with $w \in V$ such that the restriction of $f$ to $V$ is holomorphic at $w$. Then $\mathbf{E}(D, \Omega)$ is dense in $A(D, \Omega)$.

These examples point to the fact that there is a vast range of domains for which only Theorem 3 is applicable.

Let $D_{1} \subset \mathbb{C}^{n}$ and $D_{2} \subset \mathbb{C}^{m}(m \geq n \geq 1)$ be domains. A holomorphic map $f: D_{1} \rightarrow D_{2}$ is called an immersion if the rank of $f^{\prime}(z)$ is $n$ at each point $z$ of $D_{1}$, and it is called an embedding if it is a 1-1 proper holomorphic map which is also an immersion. Propositions 1 and 2 below (which we assume are well known) are merged into the proofs of Theorems 1, 2, 3 to obtain a proper holomorphic immersion for $M=2 N$ and an embedding when $M \geq 2 N+1$. A strong result of this type, using different methods, can be found in [G] where a proper holomorphic immersion is constructed from $\Delta$ to a general convex domain in $\mathbb{C}^{M}, M \geq 2$. This map is an embedding when $M \geq 3$.

Proposition 1 is designed to produce both $1-1$ and tangency (that is, $D^{\alpha} F(P)$ $=D^{\alpha} f(P)$ for all $\left.|\alpha| \leq k\right)$ in the same proper map.

Proposition 1. Let $N \geq 1, M \geq 2 N+1, D \subset \mathbb{C}^{N}$ be open and $f: D \rightarrow \mathbb{C}^{M}$ be a $C^{1}$ map. Also let $\varepsilon>0, R>0, k \geq 0$ be an integer, and $P \in \mathbb{C}^{N}$. Then there exists a holomorphic polynomial $g: \mathbb{C}^{N} \rightarrow \mathbb{C}^{M}$ such that $f+g: D \rightarrow \mathbb{C}^{M}$ is one to one, $|g|<\varepsilon$ on $R B^{N}$, and $D^{\alpha} g(P)=0$ for all $\alpha \in \mathbf{N}^{N},|\alpha| \leq k$.

Proposition 2. (1) Let $N \geq 1, M \geq 2 N, D \subset \mathbb{C}^{N}$ be open and $f: D \rightarrow \mathbb{C}^{M}$ be holomorphic. Also let $\varepsilon>0, R>0$, and $P \in \mathbb{C}^{N}$. Then there exists a holomorphic affine map $T: \mathbb{C}^{N} \rightarrow \mathbb{C}^{M}$ such that $f+T: D \rightarrow \mathbb{C}^{M}$ is an immersion, $T(P)=0$, and $|T(z)|<\varepsilon$ for all $z \in R B^{N}$.

(2) Let $M \geq N \geq 1, D \subset \mathbb{C}^{N}$ be a domain, $K \subset D$ be compact and $f: D \rightarrow \mathbb{C}^{M}$ be an immersion. There exists then $\varepsilon>0$ such that if $g: D \rightarrow \mathbb{C}^{M}$ 
is holomorphic and $|g|<\varepsilon$ on $D$ then for every $z \in K: \operatorname{Rank}\left((f+g)^{\prime}(z)\right)=N$.

It remains an open question whether Theorem 1 holds in lower codimension. The condition $M \geq \max \{2 N, 5\}$ results from Lemma 2 and has its roots in Lemma 7. There seems to be an unyielding resistance to any attempt to lower the codimension, and several distinct proofs of Lemma 2 (one of which the author includes in this paper) lead to this codimension.

A strong embedding result is proved in [EG] where a Stein manifold of dimension $N$ is embedded in $\mathbb{C}^{M}$ for the smallest integer $M>(3 N+1) / 2$. Note that the target domain in this result cannot be a general pseudoconvex domain or even the unit ball. Recall that there is no proper holomorphic map from $\mathbb{C}^{N}$ or from $\Delta^{N}$ to $B^{M}$ for any $M, N \geq 2$.

Now we will outline briefly some fundamental ideas of our main lemmas, which are Lemmas 1,2 . As before, $\rho$ is a smooth plurisubharmonic exhaustion function of the target domain $\Omega$. In both lemmas we seek a holomorphic correction function $h$ such that for $z$ on an open subset of the boundary of $D, \rho(f(z)+h(z))$ is greater than $\rho(f(z))$. It turns out that we must look separately at the case where $f(z)$ is in a small neighborhood of a critical point of $\rho$. This is done in Lemma 2. At the end of each of the lemmas, the local correction function $h$ is globalized, by a $\bar{\partial}$ application, to a global (controlled on all of $\bar{D})$ correction function $g$.

To estimate $\rho(f(z)+h(z))-\rho(f(z))$ we look at the Taylor expansion of $\rho$ at $f(z)$ up to the second order. We get that

$$
\begin{aligned}
\rho(f(z)+h(z))-\rho(f(z))= & 2 \operatorname{Re}(h(z), \bar{D} \rho(f(z)))+H_{f(z)}(h(z) \\
& +\operatorname{Re} P_{f(z)}(h(z), h(z))+o\left(|h(z)|^{2}\right),
\end{aligned}
$$

where

$$
\begin{gathered}
P_{w}(v, u)=\sum_{1 \leq j, k \leq M} D_{j} D_{k} \rho(w) v_{j} u_{k}, \\
H_{w}(v)=\sum_{1 \leq j, k \leq M} D_{j} \bar{D}_{k} \rho(w) v_{j} \bar{v}_{k} .
\end{gathered}
$$

Let us now suppose that in Lemma 1 we have (instead of $h$ ) a "conventional" correction function $h_{1}$ (of the type that appears in previous constructions) which is bounded from above and below and almost perpendicular to $\bar{D} \rho \circ f$. That is, the term $\left(h_{1}(z), \bar{D} \rho(f(z))\right)$ is marginal. Suppose also that $h_{1}$ is small enough so that the term $o\left(\left|h_{1}(z)\right|^{2}\right)$ is insignificant. Then when we look at the Taylor expansion above we find a positive contribution to $\rho\left(f(z)+h_{1}(z)\right)-\rho(f(z))$, in the term $H_{f(z)}\left(h_{1}(z)\right)$ and a major obstacle to this "positiveness" in the term $\operatorname{Re}\left(P_{f(z)}\left(h_{1}(z), h_{1}(z)\right)\right)$. To solve this problem a new type of correction function, called $h_{2}$, is added to $h_{1}$ (thus $h=h_{1}+h_{2}$ ) so that the term $\left(h_{2}(z), \bar{D} \rho(f(z))\right)$ will cancel the term $P_{f(z)}\left(h_{1}(z), h_{1}(z)\right)$. The function $h_{2}$ is much smaller (at each point) than the function $h_{1}$ and therefore it has a very small effect on the second order terms $P$ and on $H$ and on the error term $o()$. This plan succeeds due to the assumption that the size of $\bar{D} \rho(f(z))$ is bounded from below by a constant. Only one codimension is needed for this to work. 
In Lemma $2, f(z)$ is assumed to be in a small ball centered in a nondegenerate critical point. Here too $h=h_{1}+h_{2}$, where the function $h_{1}$ is similar to the one of Lemma 1. The difference from Lemma 1 is mainly in the construction and role of $h_{2}$. Here $\bar{D} \rho \circ f$ is too small for the trick of Lemma 1 to work. To accomplish the goal of compensating for the term $P\left(h_{1}, h_{1}\right)$, the function $h_{2}$ is made almost perpendicular to $h_{1}$ in order to increase the size of the positive hessian $H$. At the same time it is made "neutral" to the term $P$. Thus, by application of Lemma $7, h_{2}$ is constructed so that $\left(h_{1}(z), h_{2}(z)\right)$, $P_{f(z)}\left(h_{1}(z), h_{2}(z)\right), P_{f(z)}\left(h_{2}(z), h_{2}(z)\right)$ are almost null. The size of $h_{2}$ needs to be very large when $P\left(h_{1}, h_{1}\right)$ is large, so that the positive term $H(h, h)$ can overwhelm the term $P\left(h_{1}, h_{1}\right)$. Therefore $h_{2}$ here might have a larger size than $h_{1}$. Due to a "shortage" of codimensions we must do with an $h_{2}$ that is only "half" perpendicular to $\bar{D} \rho \circ f$.

We will now discuss the organization of our proof. After the main lemmas (Lemmas 1,2) are formulated, Propositions 1 and 2 are proven. We then present a few lemmas of common knowledge and some standard (and less standard) definitions that are applied in the proofs of the theorems and the main lemmas. Lemmas 3, 4 below are used in all constructions of low codimensional proper holomorphic maps (including ours) and therefore their proofs (which are not difficult but have not previously appeared in this context) are presented. Lemmas 5-7 are essential to the proof of Lemma 2 and they are dependent on Lemmas 3, 4.

Following this, we arrive at the proof of Theorems 1, 2 where the main lemmas are assumed. The proof is broken into several parts that include Lemmas 9,10 . The last main section of the paper contains the proofs of Lemma 1 and Lemma 2. The proof of Theorem 3 appears at the end of the paper.

The term normalized-3 below indicates that the complex hessian is globally bounded from below with factor 3, that is: $H_{\rho}(z) \geq 3|z|^{2}$ (see Definition 1.5 below). Normalization is assumed here strictly for convenience during the long calculations of the proof. The small constant $1>\varepsilon_{0}>0$ (below) is defined in [D1, Lemma 1] for $D=B^{N}$; and it depends only on the dimension $N$. In the general case it depends on the domain $D$.

Lemma 1. Let $2 \leq N, M \geq N+1, \Omega \subset \mathbb{C}^{M}$ be a domain, $\rho: \Omega \rightarrow \mathbb{R}$ be a normalized-3 $C^{2}$-smooth plurisubharmonic function, $D \subset \subset \mathbb{C}^{N}$ be a strongly pseudoconvex domain with $C^{\infty}$ boundary, and let $P \in D, z_{0} \in b D, k \geq 0$. Take also $K_{1} \subset \Omega$, a compact subset that does not contain critical points of $\rho$. There then exist constants $1>\gamma_{0}>0, C>1$, that depend only on $K_{1}, \rho, \Omega$, and a neighborhood of $z_{0}, U \subset b D$, that depends only on $z_{0}, D, P, k$ such that the following hold:

For a given $f \in A(D, \Omega), K \subset D$ compact, $\varepsilon>0$ and continuous $\gamma: b D \rightarrow$ $\left(0, \gamma_{0}\right]$ there exists $g \in A^{\infty}\left(D, \mathbb{C}^{M}\right)$ such that for all $z \in \bar{D}$ :

(a) $f(z)+g(z) \in \Omega$,

(b) $C \cdot|g(z)|^{2}+\varepsilon>\rho((f+g)(z))-\rho(f(z))>|g(z)|^{2}-\varepsilon$,

(c) if $z \in U \cap f^{-1}\left(K_{1}\right):|g(z)|>\varepsilon_{0} \gamma(z)$,

(d) if $z \in b D:|g(z)|<\left(\varepsilon_{0}\right)^{-1} \gamma(z)$,

(e) if $z \in K:|g(z)|<\varepsilon$,

(f) when $\alpha \in \mathbf{N}^{N}$ and $|\alpha| \leq k: D^{\alpha} g(P)=0$.

(The notation $A^{\infty}\left(D, \mathbb{C}^{M}\right)$ stands for the maps in $A\left(D, \mathbb{C}^{M}\right)$ that are $C^{\infty}$. smooth in $\bar{D}$.) 
Lemma 2. Let $2 \leq N, M \geq \max \{2 N, 5\}, \Omega \subset \mathbb{C}^{M}$ be a domain, $\rho: \Omega \rightarrow \mathbb{R}$ be a normalized-3 plurisubharmonic function, and let $D \subset \subset \mathbb{C}^{N}$ be a strongly pseudoconvex domain with $C^{\infty}$ boundary. Take $P \in D, z_{0} \in b D, k \geq 0$. If $w_{0}$ is an isolated critical point of $\rho$ then there exists $1>\alpha_{0}>0$, a ball $G$ centered at $w_{0}$ (both of which depend only on $w_{0}, \rho, \Omega$ ) and a neighborhood $U \subset b D$ of $z_{0}$ that depends only on $z_{0}, D, P, k$ such that the following hold:

For a given $f \in A(D, \Omega), K \subset D$ compact, $\varepsilon>0$, and $\alpha_{0} \geq \alpha>0$, there exists $g \in A^{\infty}\left(D, \mathbb{C}^{M}\right)$ such that:

(a) for all $z \in \bar{D}: f(z)+g(z) \in \Omega$,

(b) when $z \in b D: \rho((f+g)(z))-\rho(f(z))>\alpha|g(z)|^{4}-\varepsilon$,

(c) when $z \in \bar{D}: \rho((f+g)(z))-\rho(f(z))>-\alpha$,

(d) for all $z \in U \cap f^{-1}(G):|g(z)|>\varepsilon_{0} \cdot \alpha$,

(e) for all $z \in K:|g(z)|<\varepsilon$,

(f) when $\beta \in \mathbf{N}^{N}$ and $|\beta| \leq k: D^{\beta} g(P)=0$.

The lemma is true with the weaker assumption $M \geq N+1$ in the case that $w_{0}$ is a strict local minimum point.

\section{Preliminary proofs}

Introduction to the proof of Proposition 1. We will assume, without loss of generality, that $P=0$. The next sublemma is the first step.

Sublemma 1. Let $N, n \geq 1, M \geq 2 N+1, D \subset \mathbb{C}^{N}$ be open and $f: D \rightarrow \mathbb{C}^{M}$ be a $C^{1}$ map. If $v \in \mathbb{C}^{N} \backslash 0$ and $\varepsilon>0$ then there exists $y \in \varepsilon B^{M}$ such that when we define $(z \in D): F(z)=f(z)+(z, v)^{n} \cdot y$ then for all $z, w \in D$ we have: $F(z)=F(w)$ iff $(z, v)^{n}=(w, v)^{n}$ and $f(z)=f(w)$.

Proof. Elements of $\mathbb{C}^{N} \times \mathbb{C}^{N}$ will be denoted by $\langle z, w\rangle$ where $z, w \in \mathbb{C}^{N}$. Define $W=\left\{\langle z, w\rangle \in D \times D:(z, v)^{n} \neq(w, v)^{n}\right\}$; then $W$ is an open subset of $\mathbb{C}^{N} \times \mathbb{C}^{N}$. For $\langle z, w\rangle \in W$ let

$$
h(\langle z, w\rangle)=(f(z)-f(w)) /\left((w, v)^{n}-(z, v)^{n}\right) ;
$$

then $h: W \rightarrow \mathbb{C}^{M}$ is a $C^{1}$ map. Since $M>2 N$ then $h(W)$ is of Lebesgue measure 0 in $\mathbb{C}^{M}$. There therefore exists $y \in \varepsilon B^{M}$ such that $y \notin h(W)$. Define (for $z \in D$ ) $F(z)=f(z)+(z, v)^{n} y$. Now take $z, w \in D$ and assume $F(z)=F(w)$. If $(z, v)^{n} \neq(w, v)^{n}$ then $\langle z, w\rangle \in W$ and since $y=$ $(f(z)-f(w)) /\left((w, v)^{n}-(z, v)^{n}\right)$ we have a contradiction to the choice of $y$. Thus $(z, v)^{n}=(w, v)^{n}$ and therefore $f(z)=f(w)$.

Sublemma 1 is applied in the proof of the following.

Sublemma 2. Let $N, n \geq 1, M \geq 2 N+1, m=N \cdot n, \varepsilon>0, D \subset \mathbb{C}^{N}$ be open and $f: D \rightarrow \mathbb{C}^{M}$ be a $C^{1}$ map. Then there exist $v_{1}, \ldots, v_{m} \in$ $B^{N}, y_{1}, \ldots, y_{m} \in \varepsilon \cdot B^{M}$ such that when we define $(z \in D): F(z)=f(z)+$ $\sum_{1 \leq j \leq m}\left(z, v_{j}\right)^{n} \cdot y_{j}$ then for all $z, w \in D:$ we have:

$$
F(z)=F(w) \text { implies } f(z)=f(w) \text { and } w=\lambda z \text { where } \lambda \in \mathbb{C}, \lambda^{n}=1 \text {. }
$$

Proof. Take $a_{1}, \ldots, a_{m} \in \mathbb{C}$ mutually different where $0<\left|a_{i}\right|<1 / N(1 \leq$ $i \leq m)$ and let $v_{i}=\left(a_{i},\left(a_{i}\right)^{2}, \ldots,\left(a_{i}\right)^{N}\right)$; then when $S \subset\{1, \ldots, m\}$ and $|S|=N$, the set of vectors $\left\{v_{i}: i \in S\right\}$ is a basis of $\mathbb{C}^{N}$. We will define now, by induction, $C^{1}$ maps $f_{0}, \ldots, f_{m}: D \rightarrow \mathbb{C}^{M}$. First let $f_{0}=f$, then take $1 \leq j \leq m$ and assume that the $C^{1}$ map $f_{j-1}: D \rightarrow \mathbb{C}^{M}$ is defined. 
By Sublemma 1 there exists $y_{j} \in \varepsilon B^{M}$ such that when we inductively define $(z \in D): f_{j}(z)=f_{j-1}(z)+\left(z, v_{j}\right)^{n} \cdot y_{j}$ then for all $z, w \in D: f_{j}(z)=f_{j}(w)$ implies: $f_{j-1}(z)=f_{j-1}(w)$ and $\left(z, v_{j}\right)^{n}=\left(w, v_{j}\right)^{n}$. Proceed until $j=m$ is reached.

Now take $z, w \in D$ such that $f_{m}(z)=f_{m}(w)$. Then we can see by reverse induction that $f_{j}(z)=f_{j}(w)$ for all $0 \leq j \leq m$ and $\left(z, v_{j}\right)^{n}=\left(w, v_{j}\right)^{n}$ for all $1 \leq j \leq m$. Therefore for every $1 \leq j \leq m$ there exists $\lambda_{j} \in \mathbb{C}$ with $\left(\lambda_{j}\right)^{n}=1$ such that: $\lambda_{j} \cdot\left(z, v_{j}\right)=\left(w, v_{j}\right)$, or, equivalently, $\left(w-\lambda_{j} z, v_{j}\right)=0$. Since there are $n$ roots to the equation $(\xi \in \mathbb{C}): \xi^{n}=1$ and $m=n \cdot N$ there must be $\lambda \in \mathbb{C}$ with $\lambda^{n}=1$ and $S \subset\{1, \ldots, m\}$ with $|S|=N$ such that for all $j \in S:\left(w-\lambda z, v_{j}\right)=0$. From the above we get now that $w=\lambda z$.

Define $F=f_{m}$; then $F(z)=f(z)+\sum_{1 \leq j \leq m}\left(z, v_{j}\right)^{n} \cdot y_{j}$ and the proof is concluded (recall that $f=f_{0}$ ).

Proof of Proposition 1. The proof is accomplished by two applications of Sublemma 2.

(1) Take $\varepsilon^{\prime}>0, m=(k+1) N$. By Sublemma 2 there exists $v_{1}, \ldots, v_{m} \in$ $B^{N}$, and $y_{1}, \ldots, y_{m} \in \varepsilon^{\prime} \cdot B^{M}$ such that when we define $\left(z \in \mathbb{C}^{N}\right): g_{1}(z)=$ $\sum_{1 \leq j \leq m}\left(z, v_{j}\right)^{k+1} \cdot y_{j}$ and $f_{1}=f+g_{1}$ then for all $z, w \in D, f_{1}(z)=f_{1}(w)$ implies $f(z)=f(w)$ and $w=\lambda z$ for some $\lambda \in \mathbb{C}$ with $\lambda^{k+1}=1$.

(2) Put $l=(k+2) N$. By Sublemma 2 there exist $w_{1}, \ldots, w_{l} \in B^{N}$ and $x_{1}, \ldots, x_{l} \in \varepsilon^{\prime} \cdot B^{M}$ such that when we define $\left(z \in \mathbb{C}^{N}\right)$ :

$$
g_{2}(z)=\sum_{1 \leq j \leq l}\left(z, w_{j}\right)^{k+2} \cdot x_{j}
$$

and $f_{2}=f_{1}+g_{2}$ then for all $z, w \in D, f_{2}(z)=f_{2}(w)$ implies $f_{1}(z)=f_{1}(w)$ and $w=\lambda z$ for some $\lambda \in \mathbb{C}$ with $\lambda^{k+2}=1$.

(3) Combining (1) and (2) we get that if $z, w \in D$ and $f_{2}(z)=f_{2}(w)$ then by (2) $f_{1}(z)=f_{1}(w)$ and $w=\lambda z$ for some $\lambda \in \mathbb{C}$ where $\lambda^{k+2}=1$. Now by (1) $w=\lambda^{\prime} z$ where $\left(\lambda^{\prime}\right)^{k+1}=1$. If $\lambda^{\prime} \neq \lambda$ then $z=w=0$; otherwise, if $\lambda^{\prime}=\lambda$ then $\lambda^{k+2}=1$ and $\lambda^{k+1}=1$ which implies that $\lambda=1$ and $z=w$. We obtain that $f_{2}$ is one to one.

Now put $g=g_{1}+g_{2}$; then $D^{\alpha} g(0)=0$ for all $\alpha \in \mathbf{N}^{N},|\alpha| \leq k$. If $\varepsilon^{\prime}>0$ was chosen small enough then we will have $|g|<\varepsilon$ on $R B^{N}$. Now let $F=f_{2}=f+g$; by (3) the proof is completed.

Proof of Proposition 2. The proof of (2) is rather trivial. We begin with the proof of (1). We will call a subset $S$ of $\mathbb{C}^{M}$ a $k$-dimensional complex curve $(k \geq 0)$ if $S$ is a $C^{1}$ image of an open subset of $\mathbb{C}^{k}$. The proof of (1) results from the following simple claims:

Claim 1. Take integers $m, n, k$ where $n \geq 1, k \geq 0$, and $m>n+k$. Let $G \subset \mathbb{C}^{n}$ be open, and $\varepsilon>0$. If $f: G \rightarrow \mathbb{C}^{m}$ is $C^{1}$ and $S_{l} \subset \mathbb{C}^{m}, 1 \leq l<\infty$, are $k$-dimensional complex curves, then there exists $y \in \mathbb{C}^{m}$ where $|y|<\varepsilon$ such that $(y+f(G)) \cap\left(\bigcup_{1 \leq l<\infty} S_{l}\right)=\varnothing$. (For a proof see, for example, [D3, Sublemma 1]).

Claim 2. Let $V=M^{M \times N}(\mathbb{C})$ be the $(M \cdot N)$-dimensional vector space of $M \times N$ matrices with complex coefficients. Define: $E=\{A \in V: \operatorname{Rank}(A)<$ $N\}$ and let $k=N \cdot M-M+N-1$. Then $E$ is a finite union of $k$-dimensional complex curves. 
Proof. For matrix $A \in V$, where $A=\left(a_{i j}\right)_{1 \leq i \leq M, 1 \leq j \leq N}$, define $A_{j}$ to be the $j$ th column in $A$, which is the $M$-dimensional column vector: $A_{j}=$ $\left(a_{i j}\right)_{1 \leq i \leq M}$. Likewise for $M$-dimensional column vectors $A_{1}, \ldots, A_{N}$ where (for $1 \leq j \leq N) A_{j}=\left(a_{i j}\right)_{1 \leq i \leq M}$, define $A=\left[A_{1}, \ldots, A_{N}\right]$ to be the matrix in $V$ whose columns are $A_{1}, \ldots, A_{N}$. So, $A=\left(a_{i j}\right)_{1 \leq i \leq M, 1 \leq j \leq N}$.

For $1 \leq j \leq N$ put $E_{j}=\left\{A \in V: A_{j} \in s p\left\{A_{1}, \ldots, A_{j-1}\right\}\right\}$ and $V_{j}=\{A \in$ $\left.V: A_{j}=0\right\}$ (here $E_{1}=\left\{A \in V: A_{1}=0\right\}$ ); then it is clear that $E=\bigcup_{1 \leq j \leq N} E_{j}$. Now take $1 \leq j \leq N$ and define the holomorphic map $\varphi_{j}: V_{j} \times \mathbb{C}^{\bar{N}-1} \rightarrow$ $V$ in the following way: for $\lambda=\left(\lambda_{1}, \ldots, \lambda_{N-1}\right) \in \mathbb{C}^{N-1}$ and $A \in V_{j}$ let $\varphi_{j}(A, \lambda)=\left[A_{1}, \ldots, A_{j-1}, \sum_{1 \leq l \leq j-1} \lambda_{l} A_{l}, A_{j+1}, \ldots, A_{N}\right]$. It is apparent that $\varphi_{j}\left(V_{j} \times \mathbb{C}^{N-1}\right)=E_{j}$. Therefore (for $\left.1 \leq j \leq N\right) E_{j}$ is a $k$-dimensional curve.

Proof of $(1)$. The map $z \rightarrow f^{\prime}(z)$, where $f^{\prime}(z)=\left(\left(\partial f_{i} / \partial z_{j}\right)(z)\right)_{1 \leq i \leq M, 1 \leq j \leq N}$, is a holomorphic map from $D \subset \mathbb{C}^{N}$ to $V$. Since $E \subset V$ is a finite union of $k$-dimensional complex curves where $k=N \cdot M-M+N-1$ and since $M \geq 2 N$ then $N+k<M \cdot N=\operatorname{dim}(V)$. Thus by Claim 1 for a given $\varepsilon^{\prime}>0$ there exists $A \in V$ (where $A=\left(a_{i j}\right)_{1 \leq i \leq M, 1 \leq j \leq N}$ ) such that $\left|a_{i j}\right|<\varepsilon^{\prime}$ (for all $1 \leq i \leq M, 1 \leq j \leq N)$ and for all $z \in D: f^{\prime}(z)+A \notin E$, which means that $\operatorname{Rank}\left(f^{\prime}(z)+A\right)=N$. Define for $z \in \mathbb{C}^{N}: T(z)=A(z-P)$, where $z, P$ are viewed as column vectors. Then $T$ is a linear transformation from $\mathbb{C}^{N}$ to $\mathbb{C}^{M}$ and for every $z \in D:(f+T)^{\prime}(z)=f^{\prime}(z)+A$ and therefore $f+T$ is an immersion. By taking $\varepsilon^{\prime}$ small enough we can have $|T(z)|<\varepsilon$ for all $z \in R B^{N}$.

Proof of (2). For a given $z_{0} \in K$ let the column vectors $A_{N+1}, \ldots, A_{M} \in \mathbb{C}^{M}$ be such that the $M \times M$ matrix $\left[f^{\prime}\left(z_{0}\right), A_{N+1}, \ldots, A_{M}\right]$ (which consists of the matrix $f^{\prime}\left(z_{0}\right)$ with $M-N$ columns added to it) is regular. It follows that there exists an open nonempty ball, $B\left(z_{0}\right)$, around $z_{0}$, where $\bar{B}\left(z_{0}\right) \subset D$ and there exists $\delta\left(z_{0}\right)>0$ such that for all $z \in \bar{B}\left(z_{0}\right):\left|\operatorname{det}\left(\left[f^{\prime}(z), A_{N+1}, \ldots, A_{M}\right]\right)\right|>$ $\delta\left(z_{0}\right)$. Thus there exists $\varepsilon\left(z_{0}\right)>0$ such that if $g: D \rightarrow \mathbb{C}^{M}$ is holomorphic and $|g|<\varepsilon\left(z_{0}\right)$ on $D$ then for every $z \in \bar{B}\left(z_{0}\right):\left|\operatorname{det}\left(\left[(f+g)^{\prime}(z), A_{N+1}, \ldots, A_{M}\right]\right)\right|$ $>\delta\left(z_{0}\right) / 2$. Thus: $\operatorname{Rank}\left((f+g)^{\prime}(z)\right)=N$. Let $z_{1}, \ldots, z_{q} \in K$ be such that $B\left(z_{1}\right), \ldots, B\left(z_{q}\right)$ covers $K$. It is clear now that for

$$
\varepsilon=\min \left\{\varepsilon\left(z_{1}\right), \ldots, \varepsilon\left(z_{q}\right)\right\}
$$

if $g: D \rightarrow \mathbb{C}^{M}$ is holomorphic and $|g|<\varepsilon$ on $D$ then, for every $z \in$ $K, \operatorname{Rank}\left((f+g)^{\prime}(z)\right)=N$.

The next lemma is used in the proof of Lemma 4 which is applicable to all constructions of proper holomorphic maps in low codimension. Here it is used in the construction of the conventional correction function $h_{1}$ in Lemma 1 and Lemma 2 below. Lemma 5 is proved from Lemma 4, and together with Lemma 6 it leads to Lemma 7, which is an essential part of the construction of the more subtle correction function $h_{2}$ of Lemma 2 .

Lemma 3. Let $S=b B^{m}(m \geq 2), K \subset S, K \neq S$ be compact. Then there exists $U$, an open neighborhood of $K$ in $S$, and real analytic functions $u_{2}, \ldots, u_{m}$ : $U \rightarrow S$ such that $\left\{z, u_{1}(z), \ldots, u_{m-1}(z)\right\}$ is orthonormal for all $z \in U$.

Proof. Take $a \in B^{m}$ and define for $z \in \bar{B}^{m}$ :

$\varphi_{a}(z)=\left(a-\left((z, a) /|a|^{2}\right) \cdot a-\left(1-|a|^{2}\right)^{1 / 2}\left(z-\left((z, a) /|a|^{2}\right) \cdot a\right)\right) /(1-(z, a))$. 
Then $\varphi_{a}$ is holomorphic in $\bar{B}^{m}$ and is in $\operatorname{Aut}\left(B^{m}\right)$ and $\left(\varphi_{a}\right)^{-1}=\varphi_{a}$ (see [Ru, pp. 25-28]).

(1) Put $\varphi=\varphi_{a}$. We will make use of the fact that if $z \in S$ and $u \in z^{\perp}$ then $\varphi^{\prime}(z) \cdot u \in \varphi(z)^{\perp}$. To obtain this, note that when $w, v \in \mathbb{C}^{M}$ and $(v, a) \neq 1,(w, a) \neq 1$ then $(v, w)=1$ implies $(\varphi(v), \varphi(w))=1$ (see $[\mathrm{Ru}$, p. 28]). Thus for $t>0:((\varphi(z+t u)-\varphi(z)) / t, \varphi(z))=0$. If we let $t \rightarrow 0$ we obtain $\left(\varphi^{\prime}(z) \cdot u, \varphi(z)\right)=0$.

(2) Let $v \in S, \delta>0$ be such that $B(v, 2 \delta) \cap K=\varnothing$ and put $U=S \backslash \bar{B}(v, \delta)$ and $G=S \cap B(v, \delta)$. Now if we take $1>r>0$ close enough to 1 then $\varphi_{r v}(U) \subset G$. To see this take $z \in U$ and put $z=w+\lambda v$ where $\lambda \in \mathbb{C}$ and $w \perp v$. Then:

\section{Since:}

(3) $\varphi_{r v}(z)=\left((r-\lambda) v-\left(1-r^{2}\right)^{1 / 2} w\right) /(1-\lambda)$.

(4) $2|1-\lambda| \geq 2-2 \operatorname{Re}(\lambda)=|w|^{2}+|1-\lambda|^{2}=|z-v|^{2} \geq \delta^{2}$,

we get from (3) and (4) that if $1>r>0$ was chosen close enough to 1 then $\left|\varphi_{r v}(z)-v\right|<\delta$. Assume now that $1 / 10>\delta$ and fix $r$ and $\delta$ until the end of the proof.

Let $v_{1}, \ldots, v_{m} \in \mathbb{C}^{m}$ be orthonormal where $v_{m}=v$ and define for $z \in$ $G, 1 \leq i \leq m-1: w_{i}(z)=v_{i}-\left(v_{i}, z\right) \cdot z$. Then $\left(z, w_{i}(z)\right)=0$ and $w_{1}(z), \ldots, w_{m-1}(z)$ are linearly independent.

(5) Let $\varphi=\varphi_{r v}$ and define for $z \in U, 1 \leq i \leq m-1$ :

$$
y_{i}(z)=\varphi^{\prime}(\varphi(z)) \cdot w_{i}(\varphi(z)) \text {. }
$$

Since $\varphi(z) \in G$ for $z \in U$, then $y_{1}, \ldots, y_{m-1}$ are well defined real analytic functions, and (for $z \in U) y_{1}(z), \ldots, y_{m-1}(z)$ are linearly independent.

(6) Now take $z \in U$ and let $u=\varphi(z)$. Then for $1 \leq i \leq m-1$ :

$$
\left(z, y_{i}(z)\right)=\left(\varphi(u), \varphi^{\prime}(u) \cdot w_{i}(u)\right)=0 .
$$

The last equality follows from the fact that $\left(u, w_{i}(u)\right)=0$ and from (1).

Looking at (5) and (6) we see that to complete the proof all that is left is to produce $u_{1}, \ldots, u_{m-1}$ from $y_{1}, \ldots, y_{m-1}$ through the Graham Schmidt process. We first define by induction orthogonal $x_{1}, \ldots, x_{m-1}: U \rightarrow S$. Put $x_{1}=y_{1}$. If $x_{1}, \ldots, x_{j}$ are defined (where $\left.1 \leq j<m-1\right)$ we define $(z \in U)$ :

$$
x_{j+1}(z)=y_{j+1}(z)-\sum_{1 \leq l \leq j}\left(\left(y_{j+1}(z), x_{l}(z)\right) /\left|x_{l}(z)\right|^{2}\right) \cdot x_{l}(z) .
$$

By (5), (6) the functions $x_{1}, \ldots, x_{m-1}: U \rightarrow \mathbb{C}^{m}$ are now well defined nonvanishing, real analytic and, for all $z \in U,\left\{z, x_{1}(z), \ldots, x_{m-1}(z)\right\}$ is orthogonal. Now let $u_{j}(z)=x_{j}(z) /\left|x_{j}(z)\right|$ (for all $z \in U, 1 \leq j \leq m-1$ ). Then, for all $z \in U,\left\{z, u_{1}(z), \ldots, u_{m-1}(z)\right\}$ is orthonormal and $u_{1}, \ldots, u_{m-1}$ are real analytic.

In the coming discussion a real manifold of dimension $2 n-1$ could substitute $b D$ (below), but instead of meaningless generalizations we choose a formulation suitable to our purposes.

Lemma 4. Let $1 \leq n<m, 1 \leq l \leq \infty$, and let $D \subset \mathbb{C}^{n}$ be a domain with $C^{l}$ boundary.

(1) If $\psi: b D \rightarrow b B^{m}$ is $C^{l}$ then there exists $C^{l}$ functions $u_{1}, \ldots, u_{m-1}$ : $b D \rightarrow b B^{m}$ such that for all $z \in b D:\left\{\psi(z), u_{1}(z), \ldots, u_{m-1}(z)\right\}$ is orthonormal. 
(2) Let $\psi: b D \rightarrow \mathbb{C}^{m}$ be continuous and let $\varepsilon>0$. Then there exist $C^{l}$ functions $u_{1}, \ldots, u_{m-1}: b D \rightarrow b B^{m}$ such that for all $z \in b D, 1 \leq i \leq m-$ 1: $\left\{u_{1}(z), \ldots, u_{m-1}(z)\right\}$ is orthonormal and $\left|\left(u_{i}(z), \psi(z)\right)\right|<\varepsilon$.

Proof. (1) Since $\psi(b D) \subset b B^{m}$ is compact and by the positive codimension $\psi(b D) \neq b B^{m}$, then by Lemma 3 there exists $U$, an open subset of $b B^{m}$, such that $\psi(b D) \subset U$ and there are real analytic functions $v_{1}, \ldots, v_{m-1}: U \rightarrow b B^{m}$ for which $\left\{w, v_{1}(w), \ldots, v_{m-1}(w)\right\}$ is orthonormal for all $w \in U$. For $z \in$ $b D$ put $u_{i}(z)=v_{i}(\psi(z))$; then $u_{1}, \ldots, u_{m-1}: b D \rightarrow b B^{m}$ are $C^{l}$ functions and $\left\{\psi(z), u_{1}(z), \ldots, u_{m-1}(z)\right\}$ is orthonormal when $z \in b D$.

(2) Let $\psi^{\prime}: b D \rightarrow \mathbb{C}^{m}$ be a $C^{l}$ function such that $\left|\psi^{\prime}(z)-\psi(z)\right|<\varepsilon / 2$ for all $z \in b D$. Take $y \in(\varepsilon / 2) \cdot B^{m}$ where $y \notin \psi^{\prime}(b D)$ and put for $z \in b D: \varphi(z)=$ $\psi^{\prime}(z)-y$. Then for some $C>0: C>|\varphi(z)|>1 / C$ for all $z \in b D$. It follows now from (1) that there exist $C^{l}$ functions $u_{1}, \ldots, u_{m-1}: b D \rightarrow b B^{m}$ such that $\left\{\varphi(z), u_{1}(z), \ldots, u_{m-1}(z)\right\}$ is orthogonal for all $z \in b D$. Thus when $z \in$ $b D:\left|\left(u_{i}(z), \psi(z)\right)\right|<\left|\left(u_{i}(z), \psi^{\prime}(z)\right)\right|+\varepsilon / 2 \leq\left|\left(u_{i}(z), \varphi(z)\right)\right|+\left|\left(u_{i}(z), y\right)\right|<\varepsilon$.

Lemma 5. Let $1 \leq n, r, n+r \leq k, k \leq m, 1 \leq l \leq \infty, \varepsilon>0$, and let $D \subset \mathbb{C}^{n}$ be a domain with $C^{l}$ boundary. Take arbitrary continuous functions: $\psi_{1}, \ldots, \psi_{r}: b D \rightarrow \mathbb{C}^{m}$ and let $u_{1}, \ldots, u_{k}: b D \rightarrow b B^{m}$ be $C^{l}$ functions such that $\left\{u_{1}(z), \ldots, u_{k}(z)\right\}$ are orthonormal for all $z \in b D$. Define $V(z)=s p\left(u_{1}(z), \ldots, u_{k}(z)\right)$ for all $z \in b D$. There exist then $C^{l}$ functions $v_{1}, \ldots, v_{k-r}: b D \rightarrow b B^{m}$ such that for all $z \in b D, 1 \leq i \leq k-$ $r, 1 \leq j \leq r: v_{i}(z) \in V(z)$ and $\left\{v_{1}(z), \ldots, v_{k-r}(z)\right\}$ are orthonormal and $\left|\left(v_{i}(z), \psi_{j}(z)\right)\right|<\varepsilon$.

Proof. By the proof of Lemma 4(2) we can assume without loss of generality that the functions $\psi_{1}, \ldots, \psi_{r}$ are $C^{l}$ smooth. The lemma will be proven by induction on $r$, where Lemma 4 is applied.

(1) Assume first that $r=1$. Define for $z \in b D, 1 \leq j \leq k: s_{j}(z)=$ $\left(\psi_{1}(z), u_{j}(z)\right)$ and $s(z)=\left(s_{1}(z), \ldots, s_{k}(z)\right)$. Then $s: b D \rightarrow \mathbb{C}^{k}$ is a $C^{l}$ function. By Lemma 4 there exist $C^{l}$ functions $w_{1}, \ldots, w_{k-1}: b D \rightarrow \mathbb{C}^{k}$ such that for all $z \in b D, 1 \leq i \leq k-1:\left\{w_{1}(z), \ldots, w_{k-1}(z)\right\}$ is orthonormal and $\left|\left(w_{i}(z), s(z)\right)\right|<\varepsilon$. For $z \in b D$ and $1 \leq i \leq k-1$ put $w_{i}(z)=$ $\left(w_{i 1}(z), \ldots, w_{i k}(z)\right)$ and $v_{i}(z)=\sum_{1 \leq j \leq k} w_{i j}(z) \cdot u_{j}(z)$. Then $v_{1}, \ldots, v_{k-1}$ : $b D \rightarrow \mathbb{C}^{m}$ are $C^{l}$ and for all $z \in b D: v_{1}(z), \ldots, v_{k-1}(z) \in V(z)$ are orthonormal in the inner product of $\mathbb{C}^{m}$. Now for $z \in b D$ and $1 \leq i \leq$ $k-1:\left|\left(v_{i}(z), \psi_{1}(z)\right)\right|=\left|\sum_{1 \leq j \leq k} w_{i j}(z) \cdot\left(u_{j}(z), \psi_{1}(z)\right)\right|=\left|\left(w_{i}(z), s(z)\right)\right|<\varepsilon$, where the last inner product is in $\mathbb{C}^{k}$.

(2) We will now prove the lemma with the inductive assumption that it holds for $r-1$. By this assumption there exist $C^{l}$ functions $y_{1}, \ldots, y_{k-r+1}: b D \rightarrow$ $b B^{m}$ such that for all $z \in b D, 1 \leq i \leq k-r+1,1 \leq j \leq r-1: y_{i}(z) \in$ $V(z)=s p\left(u_{1}(z), \ldots, u_{k}(z)\right)$ and $\left\{y_{1}(z), \ldots, y_{k-r+1}(z)\right\}$ are orthonormal and $\left|\left(y_{i}(z), \psi_{j}(z)\right)\right|<\varepsilon / k$. For $z \in b D$ let $U(z)=s p\left(y_{1}(z), \ldots, y_{k-r+1}(z)\right)$. Note that when $z \in b D$ and $v \in U(z),|v|=1$, then $\left|\left(v, \psi_{j}(z)\right)\right|<\varepsilon$ for all $1 \leq j \leq r-1$. Now by (1) there exist $C^{l}$ functions $v_{1}, \ldots, v_{k-r}: b D \rightarrow \mathbb{C}^{m}$ such that for all $z \in b D: v_{1}(z), \ldots, v_{k-r}(z) \in U(z) \subset V(z)$ are orthonormal and for $z \in b D$ and $1 \leq i \leq k-r:\left|\left(v_{i}(z), \psi_{r}(z)\right)\right|<\varepsilon$. By the above, for $z \in b D$ and $1 \leq i \leq k-r, 1 \leq j \leq r-1:\left|\left(v_{i}(z), \psi_{j}(z)\right)\right|<\varepsilon$. The proof is thus completed. 


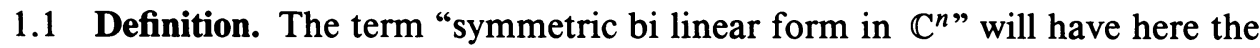
common meaning. That is, a function $\mathbf{P}: \mathbb{C}^{n} \times \mathbb{C}^{n} \rightarrow \mathbb{C}$ such that $\mathbf{P}(v, w)=$ $\mathbf{P}(w, v)$ and $\mathbf{P}\left(\lambda_{1} v_{1}+\lambda_{2} v_{2}, w\right)=\lambda_{1} \mathbf{P}\left(v_{1}, w\right)+\lambda_{2} \mathbf{P}\left(v_{2}, w\right)$ for all $v, w, v_{1}, v_{2}$ $\in \mathbb{C}^{n}, \lambda_{1}, \lambda_{2} \in \mathbb{C}$. We associate with $\mathbf{P}$ the $n \times n$ matrix $\mathbf{P}^{*}$ for which $\mathbf{P}(v, w)=\left(v, \mathbf{P}^{*} \bar{w}\right)$ where $(, \quad)$ is the standard inner product of $\mathbb{C}^{n}$ and for $w=\left(w_{1}, \ldots, w_{n}\right) \in \mathbb{C}^{n}: \bar{w}=\left(\bar{w}_{1}, \ldots, \bar{w}_{n}\right)$. For brevity's sake we sometimes write $\mathbf{P}^{2}(v)$ instead of $\mathbf{P}(v, v)$.

We now need the following fact from elementary linear algebra:

Lemma 6. Let $m \geq 2$ and $k \leq m / 2$ and let $\mathbf{P}$ be a symmetric bi-linear form in $\mathbb{C}^{m}$, then there exists a complex subspace $V \subset \mathbb{C}^{m}$ of dimension $k$ such that for all $v \in V: \mathbf{P}(v, v)=0$.

Proof. We will have $m$ (and $\mathbf{P}$ ) fixed and prove it by induction on $k$. First it is shown for $k=1$.

(1) If $u, v \in \mathbb{C}^{m}$ are linearly independent then there exists $w \in s p(u, v), w$ $\neq 0$ such that $\mathbf{P}(w, w)=0$. To see this take $w=u$ when $\mathbf{P}(u, u)=0$ and otherwise for $\lambda \in \mathbb{C}$ put $u_{\lambda}=v+\lambda u$. Then $\mathbf{P}\left(u_{\lambda}, u_{\lambda}\right)=\mathbf{P}(v, v)+2 \lambda \mathbf{P}(u, v)+$ $\lambda^{2} \mathbf{P}(u, u)$. For some $\lambda \in \mathbb{C}: \mathbf{P}\left(u_{\lambda}, u_{\lambda}\right)=0$. We then have $w=u_{\lambda}$.

(2) The lemma will be proven now with the assumption that it holds for $k-1$. There then exists a subspace $U \subset \mathbb{C}$ of dimension $k-1$ such that $\mathbf{P}(u, u)=0$ for all $u \in U$. Define $U^{*}=\left\{\mathbf{P}^{*}(\bar{u}): u \in U\right\}$. Then $U^{*}$ is a subspace of $\mathbb{C}^{m}$ of dimension $\leq k-1$. Now let $W=U+U^{*}$; then $\operatorname{dim}\left(W^{\perp}\right) \geq m-2(k-1) \geq 2$. It follows now from (1) that there exists $w \in W^{\perp}, w \neq 0$, so that $\mathbf{P}(w, w)=$ 0 . Now when $u \in U$ then, since $w \in\left(U^{*}\right)^{\perp}, \mathbf{P}(w, u)=\left(w, \mathbf{P}^{*} \bar{u}\right)=0$. Define $V=\operatorname{sp}(U \cup\{w\})$. Take $v \in V$. We can write $v=u+\lambda w$ for some $u \in U$ and $\lambda \in \mathbb{C}$. Therefore $\mathbf{P}(v, v)=\mathbf{P}(u, u)+2 \lambda \mathbf{P}(u, w)+\lambda^{2} \mathbf{P}(w, w)=0$. Since $\operatorname{dim}(V)=k$, the proof is completed.

Definition. For $z \in \mathbb{C}$ denote $z^{-}=(\operatorname{Re}(z)-|\operatorname{Re}(z)|) / 2$.

Lemma 7. Let $1 \leq n, 2 n \leq m, 1 \leq l \leq \infty$, let $D \subset C^{n}$ be a domain with $C^{l}$ boundary and let $\mathbf{P}$ be a symmetric bilinear form on $\mathbb{C}^{m}$. If $\psi: b D \rightarrow \mathbb{C}^{m}$ is continuous, $\varepsilon>0$ then:

(1) there exists a $C^{l}$ function $u: b D \rightarrow b B^{m}$ such that for all $z \in b D$ : $|\operatorname{Im}(u(z), \psi(z))|<\varepsilon,\left|(u(z), \psi(z))^{-}\right|<\varepsilon$ and $\mathbf{P}(u(z), u(z))=0$;

(2) if we add the assumption that $m \geq n+3$ then there exist $C^{l}$ functions $u_{1}, \ldots, u_{n}: b D \rightarrow b B^{m}$ such that for all $z \in b D, 1 \leq i \leq n:\left\{u_{1}(z), \ldots, u_{n}(z)\right\}$ is orthonormal and $\left|\left(u_{i}(z), \psi(z)\right)\right|<\varepsilon,\left|\left(u_{i}(z), u(z)\right)\right|<\varepsilon,\left|\mathbf{P}\left(u_{i}(z), u(z)\right)\right|<$ $\varepsilon$.

Proof. First, by the proof of Lemma 4(2) we can assume that $\psi$ is $C^{l}$ smooth. Now, by Lemma 6 there exists an $n$-dimensional subspace $V \subset \mathbb{C}^{m}$ where for all $v \in V \mathbf{P}(v, v)=0$. Let $T: \mathbb{C}^{m} \rightarrow V$ be the standard complex-linear projection into $V$ and let $\alpha=T \circ \psi$. Then $\alpha: b D \rightarrow V$ is a $C^{l}$ function. Since $b D$ is a real manifold of real dimension $2 n-1$ and $V$ is of real dimension $2 n$, there exists $v \in V,|v|<\varepsilon / 2$, such that $v \notin \alpha(b D)$. Define then for $z \in b D: \beta(z)=\alpha(z)-v$ and $u(z)=\beta(z) /|\beta(z)|$. Then $u: b D \rightarrow V \cap b B^{m}$ is $C^{l}$ and when $z \in b D: \mathbf{P}(u(z), u(z))=0$. Take now $z \in b D$. Then if $|\alpha(z)|<\varepsilon$ we have $\left|(u(z), \psi(z))^{-}\right|,|\operatorname{Im}(u(z), \psi(z))| \leq|(u(z), \psi(z))|=$ 


$$
\begin{aligned}
& |(u(z), \alpha(z))|<\varepsilon . \text { If }|\alpha(z)| \geq \varepsilon \text { then: } \\
& \qquad \begin{aligned}
|\operatorname{Im}(u(z), \psi(z))| & =|\operatorname{Im}(u(z), \alpha(z))|=\left|\operatorname{Im}\left(\left(|\alpha(z)|^{2}-(v, \alpha(z))\right) /|\beta(z)|\right)\right| \\
& =|\operatorname{Im}((v, \alpha(z)) /|\alpha(z)-v|)| \\
& \leq|\alpha(z)| \cdot|v| /(|\alpha(z)|-|v|) \\
& =|v| /(1-|v| /|\alpha(z)|) \leq 2|v|<\varepsilon,
\end{aligned}
\end{aligned}
$$

and

$$
\begin{aligned}
\left|(u(z), \psi(z))^{-}\right| & =\left|(u(z), \alpha(z))^{-}\right|=\left|\left(\left(|\alpha(z)|^{2}-(v, \alpha(z))\right) /|\beta(z)|\right)^{-}\right| \\
& \leq\left|((v, \alpha(z)) /|\alpha(z)-v|)^{-}\right| \\
& \leq|\alpha(z)| \cdot|v| /(|\alpha(z)|-|v|)<\varepsilon .
\end{aligned}
$$

(2) By Lemma 5 there exist $C^{l}$ functions $u_{1}, \ldots, u_{m-3}: b D \rightarrow b B^{m}$ such that for all $z \in b D, \quad 1 \leq i \leq m-3:\left\{u_{1}(z), \ldots, u_{m-3}(z)\right\}$ is orthonormal and $\left|\left(u_{i}(z), \psi(z)\right)\right|<\varepsilon,\left|\left(u_{i}(z), u(z)\right)\right|<\varepsilon,\left|\left(u_{i}(z), \mathbf{P}^{*} \bar{u}(z)\right)\right|<\varepsilon$. Since $\left|\mathbf{P}\left(u_{i}(z), u(z)\right)\right|=\left|\left(u_{i}(z), \mathbf{P}^{*} \bar{u}(z)\right)\right|<\varepsilon$, then (2) is proved.

1.2 Definition. Let $G \subset \mathbb{R}^{n}$ be open and $\rho: G \rightarrow \mathbb{R}$ be a $C^{2}$ function $w \in G$ is called a critical point of $\rho$ if $d \rho(w)=0$. We denote by $E_{\rho}$ the set of critical points of $\rho$ in $G$. A critical point $w \in G$ is said to be isolated when it has a neighborhood that contains no other critical point. When $w \in G$ define $d^{2} \rho(w)=\left(\left(\partial^{2} \rho / \partial x_{i} \partial x_{j}\right) \rho(w)\right)_{1 \leq i, j \leq n}$. We will say that $w$ is nondegenerate (with respect to $\rho$ ) if $\operatorname{det}\left(d^{2} \rho(w)\right) \neq 0$. Clearly a nondegenerate critical point is always isolated. This definition will be applied here in $\mathbb{C}^{n}$ which is identified with $\mathbb{R}^{2 n}$.

1.3 Definition. Let $\rho$ be a real $C^{2}$ function defined in an open subset $G \subset \mathbb{C}^{n}$ and define for $w \in G, v, u \in \mathbb{C}^{n}$ :

$$
\begin{gathered}
P_{\rho, w}(v, u)=\sum_{1 \leq j, k \leq n} D_{j} D_{k} \rho(w) v_{j} u_{k}, \quad\left(P_{\rho, w}\right)^{2}(v)=P_{\rho, w}(v, v), \\
H_{\rho, w}(v)=\sum_{1 \leq j, k \leq n} D_{j} \bar{D}_{k} \rho(w) v_{j} \bar{v}_{k}, \quad Q_{\rho, w}(v)=\operatorname{Re}\left(P_{\rho, w}(v)\right)+H_{\rho, w}(v) .
\end{gathered}
$$

The term $Q_{w}(v)$ is the second order Taylor term of $\rho$ around the point $w$. For our later needs, the "bilinearity" of $P_{\rho, w}$ is emphasized in this notation. When $w+v \in G$ we define:

$$
\alpha_{\rho, w}(v)=\rho(w+v)-\left(\rho(w)+2 \operatorname{Re}\left((v, \bar{D} \rho(w))+Q_{\rho, w}(v)\right)\right)
$$

Then $\alpha_{\rho, w}(v) /|v|^{2} \rightarrow 0$ when $w$ is fixed and $v \rightarrow 0$ and this convergence is uniform on compact subsets. Thus when $\tau>0$ and $K \subset G$ is compact there exists $\delta>0$ such that for all $w \in K$ and $v \in \mathbb{C}^{n},|v|<\delta:\left|\alpha_{\rho, w}(v)\right|<\tau|v|^{2}$. When $\rho$ is known we will write (respectively) $P_{w}, H_{w}, Q_{w}, \alpha_{w}$ instead of $P_{\rho, w}, H_{\rho, w}, Q_{\rho, w}, \alpha_{\rho, w}$.

1.4. We will use the fact that if $\rho$ is $C^{2}$ in an open set $G \subset \mathbb{C}^{n}$, and $X: \mathbb{R} \rightarrow \mathbb{R}$ is $C^{2}$ then for $r=X \circ \rho$ we have for all $w \in G, v \in \mathbb{C}^{n}$ :

(1) $\bar{D} r(w)=X^{\prime}(\rho(w)) \cdot \bar{D} \rho(w)$.

(2) $H_{r, w}(v)=X^{\prime}(\rho(w)) \cdot H_{\rho, w}(v)+X^{\prime \prime}(\rho(w)) \cdot|(v, \bar{D} \rho(w))|^{2}$. 
1.5 Definition. Let $\rho$ be a real $C^{2}$ function defined in a domain $\Omega \subset \mathbb{C}^{n}$.

(1) The function $\rho$ is said to be (strictly) plurisubharmonic at a point $w \in \Omega$ if for some $c \geq 0(c>0): H_{\rho, w}(v) \geq c|v|^{2}$ for all $v \in \mathbb{C}^{n}$, and it is called (strictly) plurisubharmonic if it is such at all points of $\Omega$. When there exists $c>0$ for which $H_{\rho, w}(v) \geq c|v|^{2}$ for all $w \in \Omega, v \in \mathbb{C}^{n}$, we say that $\rho$ is normalized- $c$.

(2) The function $\rho$ is called (strictly) convex at a point $w \in \Omega$ if for some $c \geq 0(c>0): Q_{\rho, w}(v) \geq c|v|^{2}$ for all $v \in \mathbb{C}^{n}$. It will be called (strictly) convex if it is such at every point of $w \in \Omega$. If for some $c>0: Q_{\rho}, w(v) \geq c|v|^{2}$ for all $w \in \Omega, v \in \mathbb{C}^{n}$, we say that $\rho$ is a normalized- $c$ convex function.

(3) In this paper the function $\rho$ is called an exhaustion function of $\Omega$ if $\{z \in \Omega: \rho(z) \leq a\} \subset \subset \Omega$ for all $a \in \mathbb{R}$.

(4) Note that by 1.4 , when $\rho$ is plurisubharmonic and $X: \mathbb{R} \rightarrow \mathbb{R}$ is $C^{2}$ where (for all $t$ ) $X^{\prime}(t), X^{\prime \prime}(t) \geq 0$ then $X \circ \rho$ is plurisubharmonic.

(5) A domain $\Omega \subseteq \mathbb{C}^{n}$ is called pseudoconvex if there exists a $C^{\infty}$ strictly plurisubharmonic exhaustion function for $\Omega$ (see [Ra, p. 63]). It is called $C^{k}$ smooth strongly pseudoconvex if it has a $C^{k}$ strictly plurisubharmonic defining function. There are numerous equivalent definitions to pseudoconvexity, many of which can be found in [Ra] and [K]. The most important one for us is that a domain is pseudoconvex iff it is a domain of holomorphy (see [Ra, pp. 48, 63, $75,226])$.

1.6. Claim. Let $\Omega \subset \subset \mathbb{C}^{n}$ be a pseudoconvex domain with $C^{2}$ boundary and $c>0$. Then $\Omega$ has a normalized- $c$ exhaustion function $\rho$ such that $\rho$ has no singularities in $\{w \in \Omega: \rho(w)>t\}$ for some $t>0$.

Proof. Define for $z \in \bar{\Omega}: \delta_{\Omega}(z)=d(z, b \Omega)$, and for $d>0: \Omega_{d}=\{z \in$ $\left.\Omega: \delta_{\Omega}(z)<d\right\}$. By [Ra, p. 96, p. 101 (E.5.9)] and 1.5(4) above there exists $d>0$ such that $r(z)=1 /\left(\delta_{\Omega}(z)\right)$ is a $C^{2}$ plurisubharmonic function in $\Omega_{d}$. For convenience we have $r(z)=0$ for $z \in \Omega \backslash \Omega_{d}$.

We will now adapt the solution of E.2.8 in [Ra, p. 66]. Take $a, b$ where $d>a>b>0$ and let $X: \mathbb{R} \rightarrow \mathbb{R}$ be a $C^{\infty}$ function where $X^{\prime}, X^{\prime \prime} \geq$ $0, X \equiv 0$ on $(-\infty, 1 / a]$, and for some real constant $A: X(t)=t+A$ for all $t \in(1 / b, \infty)$. Take $c>0$ and define for $z \in \Omega: \rho(z)=X(r(z))+c|z|^{2}$. Then (applying 1.5(4)) $\rho$ is a $C^{2}$ normalized- $c$, exhaustion function in $\Omega$ and, when $z \in \Omega_{b}, \rho(z)=1 /\left(\delta_{\Omega}(z)\right)+c|z|^{2}+A$. For $b>s>0$ small enough $\rho$ has no singularities in $\Omega_{s}$.

1.7 Definition. Let $\Omega \subseteq \mathbb{C}^{n}$ be a pseudoconvex domain and $c>0$. A function $\rho: \Omega \rightarrow \mathbb{R}$ is called a corrected normalized- $c$ exhaustion function if it is a $C^{\infty}$. smooth, normalized- $c$, plurisubharmonic exhaustion function where all points in $E_{\rho}$ are nondegenerate and when $z, w \in E_{\rho}, z \neq w$, then $|\rho(z)-\rho(w)|>1$.

Lemma 8. When $\Omega \subseteq \mathbb{C}^{n}$ is a pseudoconvex domain and $c>0$, then it has $a$ corrected normalized-c exhaustion function.

Proof. By [Ra, 2.21-2.22, pp. 64-65] there exists $r: \Omega \rightarrow \mathbb{R}$, a $C^{\infty}$-smooth, normalized- $2 c$, plurisubharmonic exhaustion function where $E_{r}$ is discrete and all points in $E_{r}$ are nondegenerate with respect to $r$. Take $w \in E_{r}$ and let $\delta_{w}>0$ be small enough so that $B_{w} \stackrel{\text { def }}{=} B\left(w, \delta_{w}\right)$ is contained in $\Omega$ and $\delta_{w}<$ $d\left(w, E_{r} \backslash\{w\}\right) / 2$. Then $\left\{B_{w}\right\}_{w \in E_{r}}$ are pairwise disjoint. Fix now $\varphi: \mathbb{C}^{n} \rightarrow \mathbb{R}$, a $C^{\infty}$ function such that $\varphi(0)=1, \bar{D} \rho(0)=0$, and $\operatorname{supp}(\varphi) \subset B^{n}$. Take 
$w \in E_{r}$; by the nondegeneracy of $w$ there exists $c_{w}>0$ such that for all $z \in B_{w}:|\bar{D} r(z)| \geq c_{w}|z-w|$. Define now $\alpha_{w}=\min \left\{\left|r\left(w^{\prime}\right)-r(w)\right|: w^{\prime} \in E_{r}\right.$, $\left.r\left(w^{\prime}\right) \neq r(w)\right\}$; then the discreteness of $E_{r}$ implies that $\alpha_{w}>0$ (put $\min \varnothing=$ 1). For each $w \in E_{r}$ take $\varepsilon_{w}>0$, where $\alpha_{w} / 2>\varepsilon_{w}$, such that when we define $\varphi_{w}=\varepsilon_{w} \cdot \varphi\left((z-w) / \delta_{w}\right)$ then for all $z, \xi \in \mathbb{C}^{n}:\left|\bar{D} \varphi_{w}(z)\right| \leq(1 / 2) c_{w}|z-w|$ and $\left|H_{\varphi_{w}, z}(\xi)\right| \leq(c / 2)|\xi|^{2}$. Since $E_{r}$ is countable we can choose $\left\{\varepsilon_{w}: w \in E_{r}\right\}$ to also be mutually distinct. Define $s(z)=r(z)+\sum_{w \in E_{r}} \varphi_{w}(z)$ for $z \in \Omega$. Then $s$ is a $C^{\infty}$ function on $\Omega$ with the following properties:

(1) take $z \in \Omega$; if $z \in B_{w}$ for some $w \in E_{r}$ then $s(z)=r(z)+\varphi_{w}(z)$; otherwise $s(z)=r(z)$;

(2) take $w \in E_{r} ;$ then $\bar{D} s(w)=0$ and $|\bar{D} s(z)| \geq(1 / 2) c_{w}|z-w|$ for all $z \in B_{w}$;

(3) $E_{s}=E_{r}$, and all points of $E_{s}$ are nondegenerate with respect to $s$;

(4) $s$ is normalized- $c$;

(5) when $z, w \in E_{s}$ and $z \neq w$ then $s(z) \neq s(w)$.

Proof. (1) This follows from $\sup \left(\varphi_{w}\right) \subset B_{w}$ for all $w \in E_{r}$.

(2) By (1) for $z \in B_{w}: \bar{D} s(z)=\bar{D} r(z)+\bar{D} \rho_{w}(z)$; therefore: $\bar{D} s(w)=0$, and $|\bar{D} s(z)| \geq|\bar{D} r(z)|-\left|\bar{D} \rho_{w}(z)\right| \geq(1 / 2) c_{w}|z-w|$.

(3) This follows from (1) and (2).

(4) Take $z \in \Omega, \xi \in \mathbb{C}^{n}$. If $z \in B_{w}$ for some $w \in E_{r}$ then by (1) $H_{s, z}(\xi)=$ $H_{r, z}(\xi)+H_{\rho_{w}, z}(\xi) \geq 2 c|\xi|^{2}-c|\xi|^{2}=c|\xi|^{2}$. Otherwise $H_{s, z}(\xi)=H_{r, z}(\xi) \geq$ $2 c|\xi|^{2}$.

(5) When $w \in E_{s}$ then $s(w)=r(w)+\varepsilon_{w}$. Take $z, w \in E_{s}$. If $r(z) \neq r(w)$ then since $\varepsilon_{z}, \varepsilon_{w}<|r(z)-r(w)| / 2: s(z) \neq s(w)$. If $r(z)=r(w)$ then since $\varepsilon_{z} \neq \varepsilon_{w}$ we have again $s(z) \neq s(w)$.

(6) Now let $\left\{a_{1}<a_{2}<\cdots<a_{k}<\cdots\right\}=\left\{s(w): w \in E_{s}\right\}$ and let $X: \mathbb{R} \rightarrow \mathbb{R}$ be a $C^{\infty}$ function such that $X^{\prime}(t), X^{\prime \prime}(t)>1$ for all $t \in \mathbb{R}, X(t) \rightarrow \infty$ as $t \rightarrow \infty$, and $X\left(a_{k+1}\right)-X\left(a_{k}\right)>1$ for all $k \geq 1$. Define $\rho=X \circ r$.

By 1.4 and (3), (4) above $\rho$ is a normalized- $c, C^{\infty}$ plurisubharmonic exhaustion function for $\Omega$ and $E_{\rho}=E_{s}=E_{r}$. By (3) and 1.4 all the points of $E_{\rho}$ are nondegenerate with respect to $\rho$. Looking at (5) and (6) we get that when $z, w \in E_{\rho}$ and $z \neq w$ then $|\rho(z)-\rho(w)|>1$.

\section{Proof of Theorems 1 and 2 through the Main lemmas}

The proof of Theorem 2 is split into the strongly and the weakly pseudoconvex cases. In the first case, the map produced is continuous on the boundary. After the next proof, Lemma 9 is derived from Lemma 1. Next Lemma 1 and Lemma 2 are combined to obtain Lemma 10, and from it and Lemma 9 Theorem 1 is proved.

Proof of Theorem 2 when $\Omega$ is a bounded $C^{2}$-smooth strongly pseudoconvex domain. (1) There exists a domain $\bar{\Omega} \subset \Omega_{1}$ and a $C^{2}$ strictly plurisubharmonic function $\rho$ on $\Omega_{1}$ such that $\Omega=\left\{z \in \Omega_{1}: \rho(z)<0\right\}$ and $\bar{D} \rho$ does not vanish on $b \Omega$. By shrinking $\Omega_{1}$ and multiplying $\rho$ by a positive constant we can assume that $\rho$ is normalized-3 in $\Omega_{1}, \rho$ is $C^{\prime 2}$ on $\bar{\Omega}_{1}$ and $\bar{\Omega}_{1} \backslash \Omega$ does not have critical points of $\rho$.

(2) For $a<0$ define $K_{a}=\{w \in \Omega: \rho(w)>a\}$. Fix now $a<0$ large enough so that there are no critical points of $\rho$ in $\bar{K}_{a}$ and fix $a<b<0$. Assume that $f(b D) \subset K_{b}$. 
(3) Let $\varepsilon_{0}=\varepsilon_{0}(D)$ be the small positive constant in Lemma 1 and let $C>$ $1,1>\gamma_{0}>0$, be the constants in the statement of Lemma 1 with respect to $\bar{K}_{a}, \rho, \Omega_{1}$.

Fix $U_{1}, \ldots, U_{m}(m \geq 100)$ open subsets of $b D$ where $\bigcup_{1 \leq i \leq m} U_{i}=b D$, and $U_{i}(1 \leq i \leq m)$ has the properties of $U$ in Lemma $1\left(U_{1}, \ldots, U_{m}\right.$ depend only on $D, k, P)$. For an integer $n$ let $\bar{n}$ be the only integer in $\{1, \ldots, m\}$ such that $(n-\bar{n}) / m$ is an integer.

(4) A sequence uniformly converging to the desired $F$ (the proper map that approximates $f$ on $K$ ) will now be defined by induction, where Lemma 1 is applied at each stage. Define $f_{1}=f$. Let $n \geq 1$, and assume inductively that the maps $g_{1}, \ldots, g_{n-1} \in A\left(D, \mathbb{C}^{M}\right), f_{1}, \ldots, f_{n} \in A(D, \Omega)$, where $f_{n}(b D) \subset$ $K_{a}$, are defined and $f_{n}=f_{1}+g_{1}+\cdots+g_{n-1}$.

(5) Assume $\varepsilon_{0}>\varepsilon>0$ and define:

$$
\varepsilon_{n}=\left(m^{-1} \varepsilon\right)^{10 n} \cdot \min \left\{1, b-a,\left|\rho\left(f_{i}(z)\right)\right|: z \in \bar{D}, 0 \leq i \leq n\right\} .
$$

Let

$$
\gamma_{n}(z)=\min \left\{\gamma_{0},\left(\varepsilon_{0}\right)^{2} \cdot\left|\rho\left(f_{n}(z)\right)\right|^{1 / 2} /(2 C)\right\} .
$$

(6) By Lemma 1 there exists $g_{n} \in A\left(D, \mathbb{C}^{M}\right)$ such that:

(a) for all $z \in \bar{D}: f_{n}(z)+g_{n}(z) \in \Omega_{1}$,

(b) for all $z \in \bar{D}: C \cdot\left|g_{n}(z)\right|^{2}+\varepsilon_{n}>\rho\left(\left(f_{n}+g_{n}\right)(z)\right)-\rho\left(f_{n}(z)\right)>\left|g_{n}(z)\right|^{2}-\varepsilon_{n}$,

(c) for all $z \in U_{\bar{n}}:\left|g_{n}(z)\right|>\varepsilon_{0} \gamma_{n}(z)$,

(d) for all $z \in b D:\left|g_{n}(z)\right|<\left(\varepsilon_{0}\right)^{-1} \gamma_{n}(z)$,

(e) for all $z \in K:\left|g_{n}(z)\right|<\varepsilon_{n}$,

(f) when $\alpha \in \mathbf{N}^{N}$ and $|\alpha| \leq k: D^{\alpha} g_{n}(P)=0$.

Put $f_{n+1}=f_{n}+g_{n}$.

(7) We will show that the induction assumption holds now for $n+1$. We need in fact to show that $f_{n+1}(b D) \subset K_{a}$. Take $z \in \bar{D}$; then

$$
\rho\left(f_{n+1}(z)\right)-\rho\left(f_{1}(z)\right)=\sum_{1 \leq i \leq n} \rho\left(f_{i+1}(z)\right)-\rho\left(f_{i}(z)\right)>-\left(\varepsilon_{1}+\cdots+\varepsilon_{n}\right) .
$$

Thus we get from (2) and (5) that $\rho\left(f_{n+1}(z)\right)>b+a-b=a$. Note also that for $z \in b D: \quad \rho\left(f_{n+1}(z)\right)<\rho\left(f_{n}(z)\right)+C \cdot\left|g_{n}(z)\right|^{2}+\varepsilon_{n}<$ (applying (d)) $<\rho\left(f_{n}(z)\right)+C \cdot\left(\varepsilon_{0}\right)^{-2} \gamma_{n}(z)^{2}+\varepsilon_{n} \leq \rho\left(f_{n}(z)\right)+\left|\rho\left(f_{n}(z)\right)\right| / 2+\varepsilon_{n}<$ (in view of (5)) $<0$.

We conclude that for all $z \in b D: 0>\rho\left(f_{n+1}(z)\right)>a$ and therefore $f_{n+1}(b D)$ $\subset K_{a}$, and the induction assumption prevails for $n+1$.

(8) Put $B=1-\left(\gamma_{0}\left(\varepsilon_{0}\right) /(C(1+|a|))\right)^{6}$; then $1>B>0$. From the definition of $\varepsilon_{1}, \varepsilon_{2}, \ldots$ in (5) and from (b), (c) it can be derived that for all $z \in b D$ : $B \cdot\left|\rho\left(f_{n}(z)\right)\right|<\left|\rho\left(f_{n+m}(z)\right)\right|$.

(9) We obtain for $z \in b D, n \geq 1: 2|a| B^{n / m}>\left|\rho\left(f_{n}(z)\right)\right|$.

(10) It follows then from (5) and (d) that $\sum_{1 \leq n<\infty} g_{n}$ converges uniformly on $\bar{D}$ to a map, $g \in A\left(D, \mathbb{C}^{M}\right)$.

Define $F=f+g$; then $F \in A\left(D, \Omega_{1}\right)$ is a uniform limit of $\left\{f_{n}\right\}$. By (9) and the continuity of $\rho, \rho(F(z))=0$ whenever $z \in b D$ which implies that $F$ is a continuous (on the boundary) proper holomorphic map from $D$ to $\Omega$. By (e) and (5) $|F-f|<\varepsilon$ on $K$ and by (f) $D^{\alpha} F(P)=D^{\alpha} f(P)$ for $\alpha \in \mathbf{N}^{N}$, $|\alpha| \leq k$. 
Lemma 9. Let $2 \leq N, M \geq N+1, \Omega \subset \mathbb{C}^{M}$ be a pseudoconvex domain and $\rho: \Omega \rightarrow \mathbb{R}$ be a normalized-3 $C^{2}$-smooth plurisubharmonic exhaustion function on $\Omega$. Let $D \subset \subset \mathbb{C}^{N}$ be a strongly pseudoconvex domain with $C^{\infty}$ boundary and $P \in D, k \geq 0$. Take $a, b \in \mathbb{R}, a<b$, such that the set $\rho^{-1}([a, b])$ does not contain critical points of $\rho$. Then for a given $f \in A(D, \Omega)$ where $f(b D) \subset \rho^{-1}((a, \infty)), K \subset D$ compact, $1>\varepsilon>0$ there exists $g \in A\left(D, \mathbb{C}^{M}\right)$ such that:

(a) for all $z \in \bar{D}: f(z)+g(z) \in \Omega$,

(b) for all $z \in \bar{D}: \rho(f(z)+g(z))-\rho(f(z))>-\varepsilon$,

(c) for all $z \in b D: \rho(f(z)+g(z))>b$,

(d) for all $z \in K:|g(z)|<\varepsilon$,

(e) when $\alpha \in \mathbf{N}^{N}$ and $|\alpha| \leq k: D^{\alpha} g(P)=0$.

Proof. (1) Take $\infty>c>b$ such that $\rho^{-1}([a, c]) \subset \Omega$ does not contain critical points of $\rho$, define $K_{1}=\rho^{-1}([a, c])$ and let $C>1,1>\gamma_{0}>0$ be the constants in Lemma 1 associated with our $K_{1}, \rho, \Omega$.

(2) Fix $U_{1}, \ldots, U_{m}$ an open cover of $b D$ where $U_{i}(1 \leq i \leq m)$ has the properties of $U$ in Lemma 1 and $m \geq 100$. For an integer $n$ let $\bar{n}$ be the only integer in $\{1, \ldots, m\}$ such that $(n-\bar{n}) / m$ is an integer.

(3) Define the positive integer $q=\left[m \cdot(1+c-a) /\left(\varepsilon_{0} \gamma_{0}\right)\right]^{10}$, and let $a^{\prime}=$ $\min \{\rho(f(z)): z \in b D\}$; then $a^{\prime}>a$. For $n \geq 1$ put

$$
\varepsilon^{\prime}=\varepsilon \cdot \min \left\{a^{\prime}-a, c-b, 1\right\} \cdot\left(m^{-1} \varepsilon_{0}\right)^{q} .
$$

(4) We now apply Lemma 1 to define by induction a finite sequence of functions. Put $f_{1}=f$. Let $q>n \geq 1$, and assume inductively that the maps $g_{1}, \ldots, g_{n-1} \in A\left(D, \mathbb{C}^{M}\right), f_{1}, \ldots, f_{n} \in A(D, \Omega)$ are defined and $f_{n}=f_{1}+g_{1}+\cdots+g_{n-1}$.

(5) By Lemma 1 there exists $g_{n} \in A\left(D, \mathbb{C}^{M}\right)$ such that:

(a) for all $z \in \bar{D}: f_{n}(z)+g_{n}(z) \in \Omega$,

(b) for all $z \in \bar{D}: C \cdot\left|g_{n}(z)\right|^{2}+\varepsilon^{\prime}>\rho\left(\left(f_{n}+g_{n}\right)(z)\right)-\rho\left(f_{n}(z)\right)>\left|g_{n}(z)\right|^{2}-\varepsilon^{\prime}$,

(c) for all $z \in U_{\bar{n}}$ where $c \geq \rho\left(f_{n}(z)\right) \geq a:\left|g_{n}(z)\right|>\varepsilon_{0} \gamma_{0}$,

(d) for all $z \in \bar{D}:\left|g_{n}(z)\right|<\left(\varepsilon_{0}\right)^{-1} \gamma_{0}$,

(e) for all $z \in K:\left|g_{n}(z)\right|<\varepsilon^{\prime}$,

(f) when $\alpha \in \mathbf{N}^{N}$ and $|\alpha| \leq k: D^{\alpha} g_{n}(P)=0$.

Put $f_{n+1}=f_{n}+g_{n}$. In this form we define $f_{1}, \ldots, f_{q} \in A(D, \Omega)$. We will show that $g=f_{q}-f$ satisfies our lemma.

(6) Note first that when $z \in \bar{D}, 1 \leq k \leq n \leq q$ then $\rho\left(f_{n}(z)\right)-\rho\left(f_{k}(z)\right)=$ $\sum_{k+1 \leq j \leq n} \rho\left(f_{j}(z)\right)-\rho\left(f_{j-1}(z)\right)>-q \varepsilon^{\prime}$. We obtain that for all $z \in \bar{D}$ :

(i) For $q \geq n \geq 1: \rho\left(f_{n}(z)\right) \geq \rho\left(f_{1}(z)\right)-q \varepsilon^{\prime}>a^{\prime}-q \varepsilon^{\prime}>a$.

(ii) If $\rho\left(f_{n}(z)\right)>c$ for some $q \geq n \geq 1$, then: $\rho\left(f_{q}(z)\right)>\rho\left(f_{n}(z)\right)-q \varepsilon^{\prime}>b$.

(iii) $\rho\left(f_{q}(z)\right)-\rho(f(z))>-\varepsilon$.

(7) For all $z \in b D, \rho\left(f_{q}(z)\right)>b$.

Proof. By (6) we need to show (7) only in the case that $\rho\left(f_{n}(z)\right) \leq c$ for all $q \geq n \geq 1$. In this case it follows from (6), (b) and (c), that

$$
\begin{aligned}
\rho\left(f_{q}(z)\right) & >\rho\left(f_{1}(z)\right)+(q / 2 m) \cdot\left(\varepsilon_{0} \cdot \gamma_{0}\right)^{2}-q \varepsilon^{\prime} \\
& >(\operatorname{see}(3))>a+2(c-a)-q \varepsilon^{\prime}>b .
\end{aligned}
$$

Now (a) of Lemma 9 follows from 5(a); (b) follows from (6)(iii); (c) is proved in (7); (d) is a consequence of 5(e) and (3) and 5(f) imply (e) of Lemma 9. 
The next lemma is the hard part in the proof of the Theorems from the main lemmas. It enables us to completely go through a given critical point. Sections (6) and (9) deal with the problematic situation in which $f_{n}(z)$ wanders (as $n$ progresses) closer and further to a critical point.

Since we only need Lemmas 1, 2 in the proof of Lemma 10 below, it follows from the remark at the beginning of the proof of Lemma 2 and from the fact that Lemma 1 holds in one codimension, that Lemma 10 is true in one codimension $(M \geq N+1)$ when the critical point $w_{0}$ is a strict local minimum.

In the proof of Theorem 3 (at the end of the paper), it will be shown that Lemma 1 holds (where $\operatorname{dim}(\Omega) \geq N+2$ ) when $\rho$ is $(N+1)$-dimensionalplurisubharmonic, and Lemma 2 holds (where $\operatorname{dim}(\Omega)>M_{0}=\max \{2 N, 5\}$ ) when $\rho$ is $M_{0}$-dimensional-plurisubharmonic. Lemma 2 also holds when the critical point $w_{0}$ is an $(N+1)$-dimensional-convex point of $\rho$. Thus Lemma 10 holds in the case that $\rho$ is $M_{0}$-dimensional-plurisubharmonic and also in the case where $\rho$ is $(N+1)$-dimensional-plurisubharmonic in $\Omega$ and the critical point $w_{0}$ is an $(N+1)$-dimensional-convex point of $\rho$. The proof of Lemma 9 depends only on Lemma 1 and therefore it holds (when $\operatorname{dim}(\Omega) \geq N+2$ ) if $\rho$ is $(N+1)$-dimensional-plurisubharmonic.

Lemma 10. Let $2 \leq N, M \geq \max \{2 N, 5\}, \Omega \subset \mathbb{C}^{M}$ be a pseudoconvex domain $\rho: \Omega \rightarrow \mathbb{R}$ be a corrected normalized-3 exhaustion function, and let $D \subset \subset \mathbb{C}^{N}$ be a strongly pseudoconvex domain with $C^{\infty}$ boundary and $P \in D, k \geq 0$. If $w_{0}$ is a critical point of $\rho$ and $a=\rho\left(w_{0}\right)$ and $1>\mu>0$ then there exist $1 / 4>\beta>0$ such that the following holds:

For a given $f \in A(D, \Omega)$ such that $f(b D) \subset \rho^{-1}((a-\beta, \infty)), K \subset D$ compact, $1>\varepsilon>0$, there exists $g \in A\left(D, \mathbb{C}^{M}\right)$ such that:

(a) for all $z \in \bar{D}: f(z)+g(z) \in \Omega$,

(b) when $z \in \bar{D}: \rho((f+g)(z))-\rho(f(z))>-\mu$,

(c) for all $z \in b D: \rho((f+g)(z))>a+\beta$,

(d) for all $z \in K:|g(z)|<\varepsilon$,

(e) when $\alpha \in \mathbf{N}^{N}$ and $|\alpha| \leq k: D^{\alpha} g(P)=0$.

The upper bound of the constant $\beta>0$ depends only on $\Omega, \rho, w_{0}, \mu$ and clearly $\beta>0$ can be as small as we want.

Proof. This is done by several applications of Lemma 1 and Lemma 2.

(1) Fix $U_{0}, \ldots, U_{m-1}(100 \leq m)$, an open cover of $b D$ where $U_{i}(1 \leq i \leq$ $m-1)$ has the properties of $U$ in both Lemma 1 and Lemma 2 .

(2) Let $1>\alpha_{0}>0$ and $G=B\left(w_{0}, \delta\right)$ be the constant and the ball mentioned in Lemma 2 where $1>\delta>0$. Put $G_{1}=B\left(w_{0}, \delta / 3\right), G_{2}=$ $B\left(w_{0}, 2 \delta / 3\right)$ and $K_{1}=\{z \in \Omega: a-1 / 2 \leq \rho(z) \leq a+1 / 2\} \backslash G_{1}$. Let $1>\delta_{0}>$ $0, C>1$ be the constants in Lemma 1 that are associated with our $K_{1}, \rho, \Omega$ and define $\omega=\mu \cdot \alpha_{0} / 2 m, \beta=\left(\delta \varepsilon_{0} \omega \gamma_{0} / C\right)^{10}, \varepsilon^{\prime}=(\varepsilon \beta)^{10}$.

(3) Take now $f \in A(D, \Omega)$ such that $f(b D) \subset \rho^{-1}((a-\beta, \infty))$.

(4) Put $f_{0}=f$. Lemma 1 will be applied now to define by induction $f_{0}, \ldots, f_{m} \in A(D, \Omega)$ where $\rho \circ f_{n+1}$ moves past $a+\beta$ in $U_{n}$, outside of the inverse image of $G_{1}$. Let $m>n \geq 0$, and assume inductively that the maps $g_{0}, \ldots, g_{n-1} \in A\left(D, \mathbb{C}^{M}\right), f_{0}, \ldots, f_{n} \in A(D, \Omega)$ are defined and $f_{n}=f_{0}+g_{0}+\cdots+g_{n-1}$.

(5) By Lemma 1 there exists $g_{n} \in A\left(D, \mathbb{C}^{M}\right)$ such that:

(a) for all $z \in \bar{D}: f_{n}(z)+g_{n}(z) \in \Omega$, 
(b) for all $z \in \bar{D}: C \cdot\left|g_{n}(z)\right|^{2}+\varepsilon^{\prime}>\rho\left(\left(f_{n}+g_{n}\right)(z)\right)-\rho\left(f_{n}(z)\right)>\left|g_{n}(z)\right|^{2}-\varepsilon^{\prime}$,

(c) for all $z \in U_{n} \cup f_{n}^{-1}\left(K_{1}\right):\left|g_{n}(z)\right|>\varepsilon_{0} \gamma_{0}$,

(d) for all $z \in \bar{D}:\left|g_{n}(z)\right|<\left(\varepsilon_{0}\right)^{-1} \gamma_{0}$,

(e) for all $z \in K:\left|g_{n}(z)\right|<\varepsilon^{\prime}$,

(f) when $\alpha \in \mathbf{N}^{N}$ and $|\alpha| \leq k: D^{\alpha} g_{n}(P)=0$.

Put $f_{n+1}=f_{n}+g_{n}$. Now that $f_{0}, \ldots, f_{m}$ are defined let $h_{1}=f_{m}-f$.

(6) The following hold:

(i) $\left(f_{m}\right)(\bar{D}) \subset \Omega$,

(ii) for all $z \in \bar{D}$ and $m \geq n \geq l \geq 0: \rho\left(f_{n}(z)\right)-\rho\left(f_{l}(z)\right)>(n-l) \varepsilon^{\prime}$,

(iii) for all $z \in b D: \rho\left(f_{m}(z)\right)>a-2 \beta$,

(iv) for all $z \in b D$ such that $f_{m}(z) \notin G_{2}: \rho\left(f_{m}(z)\right)>a+2 \beta$,

(v) for all $z \in K:\left|h_{1}(z)\right|<m \varepsilon^{\prime}$,

(vi) when $\alpha \in N^{N}$ and $|\alpha| \leq k: D^{\alpha}\left(h_{1}\right)(P)=0$.

Proof. We need to prove only (iv). Take $z \in b D$ such that $f_{m}(z) \notin G_{2}$ and let $m>n \geq 0$ be such that $z \in U_{n}$. By (iii) $\rho\left(f_{n}(z)\right)>a-2 \beta$, and by (ii) if $\rho\left(f_{n}(z)\right) \geq a+1 / 2$ then $\rho\left(f_{m}(z)\right)>a+2 \beta$ and (iv) holds. Thus we can assume that $a+1 / 2>\rho\left(f_{n}(z)\right)>a-1 / 2$. Now if $f_{n}(z) \notin G_{1}$ then by (c), (b), (1), (2): $\rho\left(f_{m}(z)\right)>a+2 \beta$. It is left to check the case where $f_{n}(z) \in G_{1}$. Since we assumed $f_{m}(z) \notin G_{2}$ we get now that: $\left|g_{n}(z)\right|+$ $\left|g_{n+1}(z)\right|+\cdots+\left|g_{m-1}(z)\right| \geq\left|f_{m}(z)-f_{n}(z)\right|>2 \delta / 3-\delta / 3=\delta / 3$. Therefore: $\rho\left(f_{m}(z)\right)-\rho\left(f_{n}(z)\right) \geq\left|g_{n}(z)\right|^{2}+\left|g_{n+1}(z)\right|^{2}+\cdots+\left|g_{m-1}(z)\right|^{2}-m \varepsilon^{\prime}>\delta^{2} /(9 m)$. Thus, using (iii), $\rho\left(f_{m}(z)\right)>a-2 \beta+\delta^{2} /(9 m)>a+2 \beta$, and (iv) is proved.

(7) This is the second and last stage. We will apply Lemma 2 to define by induction $f_{m+1}, \ldots, f_{2 m} \in A(D, \Omega)$ where $\rho \circ f_{2 m}$ will go past $a+\beta$ in the $f_{m}$-inverse image of $G$ and from this we will show that the obstacle critical point is completely passed. Let $2 m>n \geq m$, and assume inductively that the maps $g_{m}, \ldots, g_{n-1} \in A\left(D, \mathbb{C}^{M}\right), f_{m}, \ldots, f_{n} \in A(D, \Omega)$ are defined, and $f_{n}=f_{m}+g_{m}+\cdots+g_{n-1}$.

(8) By Lemma 2 there exists $g_{n} \in A\left(D, \mathbb{C}^{M}\right)$ such that:

(a) for all $z \in \bar{D}: f_{n}(z)+g_{n}(z) \in \Omega$,

(b) when $z \in b D: \rho\left(\left(f_{n}+g_{n}\right)(z)\right)-\rho\left(f_{n}(z)\right)>\omega\left|g_{n}(z)\right|^{4}-\varepsilon^{\prime}$,

(c) when $z \in \bar{D}: \rho\left(\left(f_{n}+g_{n}\right)(z)\right)-\rho\left(f_{n}(z)\right)>-\omega$,

(d) for all $z \in U_{n-m} \cap f_{n}^{-1}(G):\left|g_{n}(z)\right|>\varepsilon_{0} \cdot \omega$,

(e) for all $z \in K:\left|g_{n}(z)\right|<\varepsilon^{\prime}$,

(f) when $\alpha \in \mathbf{N}^{N}$ and $|\alpha| \leq k: D^{\alpha} g(P)=0$.

Let $f_{n+1}=f_{n}+g_{n}$. Define $g=f_{2 m}-f$. We will show that Lemma 10 holds with our $g, \beta$. Indeed, we need to show only (c).

(9) Take $z \in b D$, and take $m>k \geq 1$ such that $z \in U_{k}$ and let $n=m+k$. If $f_{n}(z) \in G$ then (c) (of Lemma 10) follows from 8(b), 8(d). If $f_{m}(z) \notin G_{2}$ then (c) is derived from 6(iv) and 8(b). Thus assume henceforth that $f_{m}(z) \in$ $G_{2}$ and $f_{n}(z) \notin G$. We get

$$
\left|g_{m}(z)\right|+\left|g_{m+1}(z)\right|+\cdots+\left|g_{n-1}(z)\right| \geq\left|f_{n}(z)-f_{m}(z)\right|>\delta / 3 \text {. }
$$

Therefore:

$$
\begin{aligned}
\rho\left(f_{n}(z)\right)-\rho\left(f_{m}(z)\right) & \geq \omega\left(\left|g_{m}(z)\right|^{4}+\left|g_{m+1}(z)\right|^{4}+\cdots+\left|g_{n-1}(z)\right|^{4}\right)-m \varepsilon^{\prime} \\
& >\omega \cdot(\delta /(3 m))^{4} .
\end{aligned}
$$

This with 6(iii) and (2) gives: $\rho\left(f_{n}(z)\right)>a-2 \beta+(\delta /(3 m))^{4}>a+2 \beta$, and (c) is proved and so is Lemma 10. 
In the following discussion we will ignore the immersion and embedding parts of Theorem 1. These depend only on the codimension and hold whenever $M \geq 2 N$ for immersions, and when $M \geq 2 N+1$ the maps can be embeddings. Note that the following proof of Theorem 1 holds whenever Lemmas 9, 10 hold. Therefore the result of Theorem 1 is true in one codimension $(M \geq N+1)$ when $\Omega$ has a $C^{2}$ strictly plurisubharmonic exhaustion function $\rho$ that has no saddle critical points. This gives us Corollary 1 . In fact when $M \geq N+1$, $a>0$, and $f(b D) \subset \rho^{-1}(a, \infty)$ it is enough to assume that all the critical points in $\rho^{-1}(a, \infty)$ are strict local minima. This proves Theorem 2(I). By 1.6 part II of Theorem 2 follows from part I of Theorem 2 .

If we add to these considerations the remark before Lemma 10, we obtain that the result of Theorem 1 applies also to the case when $\Omega$ is $M_{0}$-dimensionalpseudoconvex, where $M_{0}=\max \{2 N, 5\}$. Similarly, the following proof of Theorem 1, with trivial modifications, holds also in the case that $\Omega$ has an $(N+1)$-dimensional-plurisubharmonic exhaustion function $\rho$ where (for some $a \in \mathbb{R})$ all the critical points of $\rho$ in the set $\{w \in \Omega: \rho(w)>a\}$ are $(N+1)$ dimensional-convex points of $\rho$ and $f(b D) \subset \rho^{-1}(a, \infty)$. Thus Theorem 3 follows from the following proof (which relies on Lemmas 1, 2, 9, 10) and the proof at the end of the paper.

The case (in Theorem 1 ) where $\Omega$ is a bounded strongly pseudoconvex domain with $C^{2}$ boundary will be dealt with, separately, at the end of the proof.

Proof of Theorem 1. At each stage in the following inductive process, Lemma 9, Lemma 10, and, when $M \geq 2 N+1$, also Proposition 1 are applied. To prove the immersion result in Theorem 1 applications of Proposition 2 are inserted into the proof in $(*)$ and $(* *)$.

(1) Let $\rho: \Omega \rightarrow \mathbb{R}$ be a corrected normalized-3 exhaustion function; then by Definition $1.7 E_{\rho}$ is countable and it can be arranged so that $E_{\rho}=\left\{w_{1}, \ldots\right.$, $\left.w_{n}, \ldots\right\}$ and if $a_{n}=\rho\left(w_{n}\right)(1 \leq n)$ then $a_{n+1}>a_{n}+1$ for all $n \geq 1$. Note that $\rho\left(w_{1}\right)=\min \{\rho(w): w \in \Omega\}$. For $n \geq 1$ put $\mu_{n}=10^{-n}$ and let $\beta_{n}, 1 / 4>\beta_{n}>0$, be the constant associated in Lemma 10 with $w_{n}, \mu_{n}, \Omega, \rho$.

We will assume that $E_{\rho}$ is infinite. When $E_{\rho}$ is finite the proof below becomes easier. In this case $E_{\rho}=\left\{w_{1}, \ldots, w_{k}\right\}$ for some $k \geq 1$. Continue then $a_{1}, \ldots, a_{k}$, by adding a sequence of real numbers $a_{k+1}, a_{k+2}, \ldots$, so that for all $1 \leq n<\infty: a_{n+1}>a_{n}+1$. The inductive process will go exactly as below until the $k$-step. After that we will omit at each inductive step the application of Lemma 10 at (5) and therefore will skip the definition of $g_{n, 1}$ (for all $n>k$ ). The rest of the proof goes unchanged except for some trivial modifications.

(2) Take $K_{1}, K_{2}, \ldots \subset D$, an infinite sequence of compact subsets, such that $K \subset K_{1}, K_{n} \subset \operatorname{int}\left(K_{n+1}\right)$ for all $\infty>n \geq 1$ and $\bigcup_{1 \leq n<\infty} K_{n}=D$.

(3) Here, as before, we define $f_{1}=f$ and for $n \geq 1$, we assume inductively that the maps $g_{1}, \ldots, g_{n-1} \in A\left(D, \mathbb{C}^{M}\right), f_{1}, \ldots, f_{n} \in A(D, \Omega)$ are defined and $f_{n}=f_{1}+g_{1}+\cdots+g_{n-1}$. Assume also that $f_{n}(b D) \subset \rho^{-1}\left(\left(a_{n}-\beta_{n}, \infty\right)\right)$ (this holds for $n=1$ ).

In first reading, or when $M<2 N$ (where $M=\operatorname{dim}(\Omega), N=\operatorname{dim}(D)$ ), the reader may skip the immersion branch of the proof $((2)$ of Theorem 1) and therefore overlook the following $(*)$ and do the same, later, with $(* *)$ below. When doing so, put for formality's sake $\sigma_{j}=1(1 \leq j \leq n)$. 
(*) When $M \geq 2 N$ merge here (for the proof of (2)), the assumption that $f_{j}: \operatorname{int}\left(K_{j}\right) \rightarrow \mathbb{C}^{M}(1 \leq j \leq n)$ is an immersion. Then (for $1 \leq j \leq n$ ) take $\sigma_{j}>0$ such that for every holomorphic $h: D \rightarrow \mathbb{C}^{M}$, where $|h|<\sigma_{j}$ on $K_{j}: \operatorname{Rank}\left(f_{j}+h\right)^{\prime}(z)=N$ for all $z \in K_{j-1}$ (see Proposition 2(2)).

(4) In the case that $M \geq 2 N+1$ we assume, in addition, that $f_{1}, \ldots, f_{n}$ are one to one and define (for $1 \leq j \leq n): \delta_{j}=\min \left\{\left|f_{j}(z)-f_{j}(w)\right|: z, w \in\right.$ $\left.K_{j-1},|z-w| \geq \varepsilon_{j-1}\right\}$; then $\delta_{j}>0$. When $M<2 N+1$ define $\delta_{1}=\cdots=$ $\delta_{n}=1$

(5) Assume $1 / 1000>\varepsilon>0$ and define: $\varepsilon_{n}=\varepsilon^{n+1} \cdot \min \left\{d\left(\bigcup_{1 \leq j \leq n} f_{j}(\bar{D}), b \Omega\right)\right.$, $\left.\delta_{1}, \delta_{2}, \ldots, \delta_{n}, \sigma_{1}, \sigma_{2}, \ldots, \sigma_{n}, \beta_{1}, \ldots, \beta_{n+1}\right\}$.

(6) By Lemma 10 there exists $g_{n, 1} \in A\left(D, \mathbb{C}^{M}\right)$, such that:

(a) for all $z \in \bar{D}: f_{n}(z)+g_{n, 1}(z) \in \Omega$,

(b) when $z \in \bar{D}: \rho\left(\left(f_{n}+g_{n, 1}\right)(z)\right)-\rho\left(f_{n}(z)\right)>-\mu_{n}$,

(c) for all $z \in b D: \rho\left(\left(f_{n}+g_{n, 1}\right)(z)\right)>a_{n}+\beta_{n}$,

(d) for all $z \in K_{n}:\left|g_{n, 1}(z)\right|<\varepsilon_{n}$,

(e) when $\alpha \in \mathbf{N}^{N}$ and $|\alpha| \leq k: D^{\alpha} g_{n, 1}(P)=0$.

(7) In view of (c) we can apply now Lemma 9. There exists $g_{n, 2} \in A\left(D, \mathbb{C}^{M}\right)$ such that:

(a) for all $z \in \bar{D}: f_{n}(z)+g_{n, 1}(z)+g_{n, 2}(z) \in \Omega$,

(b) for all $z \in \bar{D}: \rho\left(f(z)+g_{n, 1}(z)+g_{n, 2}(z)\right)-\rho\left(f(z)+g_{n, 1}(z)\right)>-\varepsilon_{n}$,

(c) for all $z \in b D: \rho\left(f(z)+g_{n, 1}(z)+g_{n, 1}(z)\right)>a_{n+1}-\beta_{n+1} / 2$,

(d) for all $z \in K_{n}:\left|g_{n, 2}(z)\right|<\varepsilon_{n}$,

(e) when $\alpha \in \mathbf{N}^{N}$ and $|\alpha| \leq k: D^{\alpha} g_{n, 2}(P)=0$.

(8) When $M \leq 2 N$ then, to prove (1) of Theorem 1, we define $g_{n}=g_{n, 1}+$ $g_{n, 2}$ and $f_{n+1}=f_{n}+g_{n}$, and now go to the next induction step (note that the induction assumption holds now for $n+1)$. In this case (the proof of $(1)$ ) the reader may proceed directly to (11).

(9) Otherwise (to prove (2) or (3)) let $\varepsilon_{n}^{\prime}>0$ be such that $\varepsilon_{n}^{\prime}<\varepsilon_{n}$ and if $w \in\left(f+g_{n, 1}+g_{n, 2}\right)(\bar{D}), v \in \mathbb{C}^{M}$, and $|w-v|<2 \varepsilon_{n}^{\prime}$, then $v \in \Omega$ and $|\rho(w)-\rho(v)|<\varepsilon_{n}$.

When not proving (2), skip the following $(* *)$ and then put for formality $g_{n, 3} \equiv 0$ and $\varepsilon_{n}^{\prime \prime}=\varepsilon_{n}^{\prime}$.

(**) For the proof of (2) (when $M \geq 2 N$ ) let $g_{n, 3}$ be a holomorphic affine map from $\mathbb{C}^{N}$ to $\mathbb{C}^{M}$ such that $\left(f_{n}+g_{n, 1}+g_{n, 2}+g_{n, 3}\right): D \rightarrow \mathbb{C}^{M}$ is an immersion, $g_{n, 3}(P)=0$, and $\left|g_{n, 3}(z)\right|<\varepsilon_{n}^{\prime}$ for all $z \in D$. Note that when $\alpha \in \mathbf{N}^{N}$ and $|\alpha| \neq 1: D^{\alpha} g_{n, 3}(P)=0$. If $M=2 N$ then put $g_{n}=g_{n, 1}+$ $g_{n, 2}+g_{n, 3}, f_{n+1}=f_{n}+g_{n}$ and proceed to (11). Now let $\varepsilon_{n}^{\prime \prime}$ be such that $\varepsilon_{n}^{\prime}>\varepsilon_{n}^{\prime \prime}>0$ and if $h: D \rightarrow \mathbb{C}^{M}$ is holomorphic and $|h|<\varepsilon_{n}^{\prime \prime}$ on $D$ then for every $z \in K_{n}: \operatorname{Rank}\left(f_{n}+g_{n, 1}+g_{n, 2}+g_{n, 3}+h\right)^{\prime}(z)=N$ (see Proposition 2(2)).

(10) When $M \geq 2 M+1$ then by Proposition 1 there exists a holomorphic polynomial $g_{n, 4}: \mathbb{C}^{N} \rightarrow \mathbb{C}^{M}$ such that $f_{n}+\sum_{1 \leq l \leq 4} g_{n, l}$ is one to one on $\bar{D}$, $\left|g_{n, 4}\right|<\varepsilon_{n}^{\prime \prime}$ on $\bar{D}$, and $D^{\alpha} g_{n, 4}(P)=0$ for all $\alpha \in \mathbf{N}^{N},|\alpha| \leq k$. In this case we define $g_{n}=\sum_{1 \leq l \leq 4} g_{n, l}$ and $f_{n+1}=f_{n}+g_{n}$. It is clear that in all three cases the respective induction assumptions hold now for $n+1$.

(11) Now (in all cases) $\left\{f_{n}\right\}_{1 \leq n<\infty}$ converges uniformly on compacta. Call its limit $F$; then $F: D \rightarrow \bar{\Omega}$ is holomorphic. It is easy to calculate that $|F-f|<\varepsilon$ on $K$ and: $D^{\alpha} F(P)=D^{\alpha} f(P)$ for all $\alpha \in \mathbf{N}^{N},|\alpha| \leq k$ (when we prove (2) 
then $D^{\alpha} F(P)=D^{\alpha} f(P)$ for all $\left.\alpha \in \mathbf{N}^{N},|\alpha| \leq k,|\alpha| \neq 1\right)$. We will show now that $F: D \rightarrow \Omega$ is proper.

(12) First, we need to show that $F(D) \subset \Omega$. Take $z \in D$ and let $n$ be large enough so that $z \in K_{n}$. Since $z \in K_{m}$ for all $m \geq n$ then by (5)-(10): $\left|F(z)-f_{n}(z)\right| \leq \sum_{\infty>m \geq n}\left|g_{m}(z)\right|<4 \sum_{\infty>m \geq n} \varepsilon_{m}<8 \varepsilon_{n}<d\left(f_{n}(z), b \Omega\right)$. It follows that $F(z) \in \Omega$.

The proof that $F$ is proper is almost the same as the one in the proof of Theorem 1 in [D3], but now, because of 6(b), the backslides of $\rho \circ f_{n}$ are larger inside $D$. However, since $\left\{\mu_{n}\right\}$ have a finite sum, this will be no hindrance.

(13) Note that when $z \in D$ and $1 \leq n<\infty$ then from 6(b), 7(b) and (9) we obtain that: $\rho(F(z)) \geq \rho\left(f_{n}(z)\right)-\sum_{1 \leq j<\infty} \mu_{j}+3 \varepsilon_{n}>\rho\left(f_{n}(z)\right)-1$.

(14) Take arbitrary $z \in b D$ and any sequence $\left\{z_{n}\right\}_{1 \leq n<\infty} \subset D$ which converges to $z$. We will show that $\lim _{n \rightarrow \infty} \rho\left(F\left(z_{n}\right)\right)=\infty$.

(15) Take arbitrary $A>0$ and let $k$ be such that $\rho\left(f_{k}(z)\right)>A+2$. There exists $r \geq 1$ such that for all $n>r: \rho\left(f_{k}\left(z_{n}\right)\right)>A+1$. By (13) for all $n>r: \rho\left(F\left(z_{n}\right)\right)>A$. Now (14) is proved and therefore $F$ is proper.

(16) To prove the immersion result in Theorem 1 (2) we have to show that if $M \geq 2 N$ and $(*),(* *)$ were applied in the induction process then the map $F$ is an immersion. Take $n \geq 1$; by the induction assumption $f_{n}$ is an immersion on $\operatorname{int}\left(K_{n}\right)$. Now for $z \in K_{n}:\left|F(z)-f_{n}(z)\right| \leq \sum_{n \leq j<\infty}\left|g_{j}(z)\right|<$ $4 \sum_{n \leq j<\infty} \varepsilon_{j}<8 \varepsilon_{n}<\sigma_{n}$. Therefore by $(*) \quad F$ is an immersion on $\operatorname{int}\left(K_{n-1}\right)$. Since this is true for all $n \geq 1, F$ is an immersion on $D$.

(17) We now show that when $M \geq 2 N+1, F$ is one to one. Take $z, w \in$ $D, w \neq z$. For $n \geq 1$ large enough, $z, w \in K_{n-1}$ and $|z-w| \geq \varepsilon_{n-1}$. By definition (4) $0<\delta_{n}<\left|f_{n}(z)-f_{n}(w)\right|$. Now $|F(z)-F(w)|>\left|f_{n}(z)-f_{n}(w)\right|-$ $\left|F(z)-f_{n}(z)\right|-\left|F(w)-f_{n}(w)\right|>($ see $(5)$ and $(16))>\delta_{n}-16 \varepsilon_{n}>0$.

The main part of the proof is completed. It is left to show that in all cases $(M<2 N, M=2 N, M>2 N) F$ can be also continuous on $\bar{D}$ when we assume that $\Omega$ is a bounded strongly pseudoconvex domain with $C^{2}$ boundary. In the beginning of the proof of Theorem 2 (the strongly pseudoconvex case) we mentioned that there exist a domain $\bar{\Omega} \subset \Omega_{1}$ and a $C^{2}$ normalized-3 plurisubharmonic function $r$ on $\Omega_{1}$ such that $\Omega=\left\{w \in \Omega_{1}: r(w)<0\right\}$ and $\Omega_{1} \backslash \Omega$ does not have critical points of $r$. Let $t<0$ be such that $\{w \in$ $\left.\Omega_{1}: r(w) \geq t\right\}$ has no critical points of $r$ and let $n_{0}$ be large enough such that $f_{n_{0}}(b D) \subset\left\{w \in \Omega_{1}: r(w)>t\right\}$ (where $\left\{f_{n}\right\}$ are defined in the proof of Theorem 1 above). The proof is completed when we apply the process of the proof of Theorem 2 (in the strongly pseudoconvex case) with the starting function $f=f_{n_{0}}$ and defining function $r$.

Proposition 2 can be inserted (when $M \geq 2 N$ ) to obtain a continuous proper holomorphic immersion. When $M \geq 2 N+1$ we insert Proposition 1 into this proof, as it was done above, to get (in addition) an injective map.

\section{Proof of Lemma 1}

Our starting point is a description of the properties of the "lattice" of peak functions developed in [D1]. We will not repeat the derivation of these properties, as this is a long process which can be found in [D1]. The set of peak functions that we are about to discuss is indexed by a finite subset of $\mathbb{Z}^{2 N-1}$ and we need the following function $\pi$ to subdivide this index set. 
3.1 For $a=\left(a_{1}, \ldots, a_{2 N-1}\right) \in \mathbb{Z}^{2 N-1}$ define $\pi(a)=\left(a_{N}+\cdots+a_{2 N-2}\right) \bmod N$. For the sake of convenience we look at the values of the function $\pi$ as integers between 1 and $N$.

The function $(r>0, z \in \bar{D}): \kappa_{r}(z)=\left(\varepsilon_{0}\right)^{d\left(z_{0}, b D\right) /\left(r \cdot N^{-5}\right)}$ will express, in the case $D=B^{N}$, the decline of the peak functions we describe below. In the general pseudoconvex case another constant takes the place of $N^{-5}$. When $r$ is known we will write: $\kappa=\kappa_{r}$. When $z \in \bar{D}$ let $z^{*}$ be the closest point to $z$ in $b D$, whenever well defined.

3.2 We will describe the set-up in [D1] for the network of peak functions that we will use. The peak functions of [D1] were developed for the ball case, but the properties (i)-(v) described below hold (with very slight modifications) for the general pseudoconvex case. This is seen when one inserts in the proof of Lemma 1 of [D1] the peak functions of [S] (or [H]). The results of this lemma (that depend mainly on the close point property (Lemma 4 of [D1]) and the peak functions) then generalize, almost automatically, to the pseudoconvex case. A parameter $d>0$ was defined in [D1], whose upper bound depends on $N$, in the ball case, and on the domain in the general pseudoconvex case. This parameter is used to define the following sets: $W=B\left(z_{0}, d\right), W^{\prime}=$ $B\left(z_{0}, 2 d\right), W^{*}=B\left(z_{0}, 1.5 d\right), U=W \cap b D, U^{*}=W^{*} \cap b D$.

Now take $\varepsilon_{1}>0$. It was shown that for $r>0$ small enough (there is no positive lower bound for $r$ ) there exists a set $\left\{z_{a}: a \in L\right\}$ where $L \subset \mathbb{Z}^{2 N-1}$ (that can be justly called a lattice), which is included in $B\left(z_{0}, d+r^{0.2}\right) \cap b D$. There exist also local peak functions, $p_{a} \in A^{\infty}(D), a \in L$, for which the following properties, (i)-(v), hold:

(i) $p_{a}$ peaks at $z_{a}$, that is: $p_{a}\left(z_{a}\right)=1$ and $1>\left|p_{a}\right|>0$ on $W^{\prime} \backslash\left\{z_{a}\right\}$,

(ii) let $\kappa=\kappa_{r}$; then for all $z \in W^{\prime}, \sum_{a \in L}\left|p_{a}(z)\right|<\left(4 \varepsilon_{0}\right)^{-1} \cdot \kappa(z)$,

(iii) define for $1 \leq i \leq N: L_{i}=\{a \in L ; \pi(a)=i\}$; when $z \in U^{*}$ there exists $i=i(z)$ such that $\left|\sum_{a \in L_{i}} p_{a}(z)\right|>4 \varepsilon_{0}$,

(iv) for all $z \in W^{\prime}, \sum_{a \in L,\left|z_{a}-z\right|>r^{0.2}}\left|p_{a}(z)\right|<\left(\varepsilon_{1}\right)^{4}$,

(v) for all $z \in W^{\prime}, \sum_{a \in L}\left|p_{a}(z)-p_{a}\left(z^{*}\right) \cdot \kappa(z)\right|<\left(\varepsilon_{1}\right)^{4}$.

The interested reader can learn from [D1] how this was derived, but for our purposes these properties will suffice. The parameter $r>0$ gives in fact the spacings (or the density) of the lattice $\left\{z_{a}: a \in L\right\}$. The final choice of $r>0$ is made in 3.7. We can take $d>0$ smaller, as small as we want, and then shrink $r>0$ accordingly to maintain (i)-(iv). In the general pseudoconvex case $d>0$ has to be small enough so that the correspondence $z \rightarrow z^{*}$ is well defined and continuous in $W^{\prime}$.

Clearly the presentation of these properties would have been less confusing and would have exactly the same meaning if $\left(\varepsilon_{1}\right)^{4}$ was replaced by $\varepsilon_{1}$ but the formulation above is tailored to simplify our calculations later.

In [D1] the sets $W, W^{\prime}$ are defined somewhat differently and the properties (i)-(v) appear in a different form but it is not hard to obtain the above from [D1]. The proof in [D1] that such a system of peak functions exists consists of two main components: the choice of the peak functions in [D1, 0], and their distribution, based on the close point principle ([D1, Lemma 3]).

Next, these peak functions are corrected so that their derivatives in the point $P$ will vanish to the $k$ order.

3.3 Let $z_{0}=\left(z_{0,1}, \ldots, z_{0, N}\right)$ and $P=\left(P_{1}, \ldots, P_{N}\right)$. Fix $1 \leq j_{0} \leq N$ 
so that $\left|z_{0, j_{0}}-P_{j_{0}}\right|>0$ and define for $z=\left(z_{1}, \ldots, z_{N}\right) \in \mathbb{C}^{N}: S(z)=$ $1.5\left(z_{j_{0}}-P_{j_{0}}\right)^{k+1} /\left(z_{0, j_{0}}-P_{j_{0}}\right)^{k+1}$. Now assume that $d>0$ was taken small enough such that $2>|S(z)|>1.2$ for all $z \in W^{\prime}$. Define for $z \in \bar{D}$ and $a \in L: q_{a}(z)=S(z) \cdot p_{a}(z)$.

The notation $l(r)=-1 / \log (r)$ will be used throughout the paper. The parameter $r>0$ must be taken small enough such that when $z, w \in \bar{D}$ and $|z-w|<-\log (r)$ then $|S(z)-S(w)|<\left(\varepsilon_{1}\right)^{4}$. The following hold then:

(i) for all $z \in W^{\prime}, \sum_{a \in L}\left|q_{a}(z)\right|<\left(2 \varepsilon_{0}\right)^{-1} \cdot \kappa(z)$,

(ii) when $z \in U^{*}$ then $\left|\sum_{a \in L_{i(z)}} q_{a}(z)\right|>4 \varepsilon_{0}$,

(iii) for all $z \in W^{\prime}, \sum_{a \in L,\left|z_{a}-z\right|>r^{0.2}}\left|q_{a}(z)\right|<2\left(\varepsilon_{1}\right)^{4}$,

(iv) for all $z \in W^{\prime}, \sum_{a \in L}\left|q_{a}(z)-q_{a}\left(z^{*}\right) \cdot \kappa(z)\right|<2\left(\varepsilon_{1}\right)^{4}$,

(v) when $a \in L, \alpha \in \mathbf{N}^{N}$ and $|\alpha| \leq k: D^{\alpha}\left(q_{a}\right)(P)=0$.

The reader will notice later that the globalization processes (in 3.15 and 4.24) are made so that for the correction function $g: D^{\alpha} g(P)=0$ for all $\alpha \in \mathbf{N}^{N},|\alpha| \leq k$. Therefore it appears to be unnecessary to define $\left\{q_{a}\right\}$ for the same purpose. However $\left\{q_{a}\right\}$ are presented here to demonstrate the fact that the proofs of Theorems 1,2 can be completely elementary in the case that $D=B^{N}$. In this ball case we can take global peak functions $\left\{p_{a}\right\}$ that have the properties $(\mathrm{i})-(\mathrm{v})$ in 3.2 globally and then the globalization process (that requires a $\bar{\partial}$ application) is not needed. These peak functions, like the ones developed in [D1], are exponents of polynomials. They can be found in an early (unpublished) version of the paper [D4] (available on request from the author).

The next stage will be the construction of the correction function $h_{1}$ and a description of its properties, in 3.10.

3.4 For $w \in \Omega$ let $\alpha_{w}=\alpha_{\rho, w}, P_{w}=P_{\rho, w}, H_{w}=H_{\rho, w}$ be as in Definition 1.3. Take $\lambda>0$ such that $\min \left\{1, d\left(K_{1}, b \Omega\right)\right\} / 2>\lambda$, and when we define $K_{2}=\left\{z \in \mathbb{C}^{M}: d\left(z, K_{1}\right) \leq \lambda\right\}$ then $K_{2}$ has no critical points of $\rho$. As mentioned in 1.3 , there exists $\delta_{0}>0$, where $\lambda>\delta_{0}$, such that for all $w \in K_{2}$ and $v \in \mathbb{C}^{M},|v|<\delta_{0}:\left|\alpha_{w}(v)\right|<|v|^{2}$. Define:

$C=4 \max \left\{\left|P_{w}(v, u)\right|,\left|H_{w}(v)\right|,|\bar{D} \rho(w)|, 1 /|\bar{D} \rho(w)|: w \in K_{2}, v, u \in b B^{m}\right\} ;$ then (by the normalization) $C>12$. Define also $C^{\prime}=\max \{|S(z)|: z \in \bar{D}\}$ and put: $\gamma_{0}=\left(\varepsilon_{0} \cdot \delta_{0} / C\right)^{100}$. Let $\gamma: b D \rightarrow\left(0, \gamma_{0}\right]$ be continuous and let $\gamma_{1}=\min \{\gamma(z): z \in b D\}$ (then $\left.\gamma_{1}>0\right)$. Assume that the given $\varepsilon>0$ (in the statement of the lemma) is so small that $\varepsilon<\left(\min \left\{\gamma_{1}, d(K, b D)\right\} /\left(1+C^{\prime}\right)\right)^{100}$. There is no loss of generality in this assumption.

3.5 Fix now $0<\varepsilon_{1}$ where

$$
\varepsilon_{1}<\varepsilon^{10} \cdot(1+\max \{|\bar{D} \rho(w)|: w \in f(\bar{D})\}+1 / d(f(\bar{D}), b \Omega))^{-10},
$$

and for all $w \in f(\bar{D}), v \in\left(\varepsilon_{1}\right)^{0.1} \cdot B^{M}:|\rho(w+v)-\rho(w)|<\varepsilon^{10}$. Assume that 3.2, 3.3 hold with this $\varepsilon_{1}$. We require that $r>0$ is chosen small enough so that $r<\left(\varepsilon_{1}\right)^{1 / \varepsilon_{1}}$ and when $z, w \in \bar{D},|z-w|<l(r)$, then:

(i) $|\bar{D} \rho(f(z))-\bar{D} \rho(f(w))|<\left(\varepsilon_{1}\right)^{2}$,

(ii) when $z, w \in b D:|\gamma(z)-\gamma(w)|<\left(\varepsilon_{1}\right)^{2}$,

(iii) for all $1 \leq i, j \leq M:\left|D_{i} D_{j} \rho(f(z))-D_{i} D_{j} \rho(f(w))\right|<\left(\varepsilon_{1}\right)^{2} / M^{2}$.

We obtain from (iii) that for all $v, u \in b B^{M}$ :

(iv) $\left|P_{f(z)}(v, u)-P_{f(w)}(v, u)\right|<\left(\varepsilon_{1}\right)^{2}$,

(v) $|f(z)-f(w)|<\left(\varepsilon_{1}\right)^{2}$.

3.6 By Lemma 4 there exist $C^{1}$ functions $u_{1}, \ldots, u_{N}: b D \rightarrow \mathbb{C}^{M}$ such that 
for every $z \in b D:\left\{u_{1}(z), \ldots, u_{N}(z)\right\}$ is orthonormal, and $\left|\left(\bar{D} \rho(f(z)), u_{i}(z)\right)\right|$ $<\left(\varepsilon_{1}\right)^{2}$. By shrinking $r>0$ further, if necessary, we can assume that when $z, w \in b D$ are such that $|z-w|<l(r)$ then for all $i=1,2, \ldots, N: \mid u_{i}(z)-$ $u_{i}(w) \mid<\left(\varepsilon_{1}\right)^{2}$.

3.7 Now define a continuous nonnegative function that gives the size of $g$ on $b D$, up to a bounded proportion and a small error term. First let $U_{1}=\{z \in$ $\left.U^{*}: d\left(z, f^{-1}\left(K_{1}\right)\right) \leq l(r)\right\}$. Let $t(z)=\gamma(z)$ when $z \in U_{1}$ and $d\left(z, b U_{1}\right) \geq$ $l(r)$. If $z \in U_{1}$ and $d\left(z, b U_{1}\right) \leq l(r)$ then put $t(z)=\gamma(z) \cdot d\left(z, b U_{1}\right) / l(r)$. When $z \in b D \backslash U_{1}$ then define: $t(z)=0$. Extend it continuously to $W^{\prime} \cup b D$; put for $z \in W^{\prime}: t(z)=t\left(z^{*}\right)$. By shrinking $r>0$ further we can assume that when $z, w \in W^{\prime},|z-w|<l(r)$, then: $|t(z)-t(w)|<\left(\varepsilon_{1}\right)^{2}$. Notice that if $z \in W^{\prime}$ and $d\left(z, U_{1}\right)<l(r)$ then $f(z) \in K_{2}$. Define $t_{a}=t\left(z_{a}\right)$ for $a \in L$.

3.8 Take $a \in L$, let $i=\pi(a)$ and define: $u_{a}=u_{i}\left(z_{a}\right), v_{a}=t_{a} \cdot u_{a}$. It can easily be calculated from $3.6,3.7$ (as in [D1, 1.14]) that for all $a, b \in L$ :

(i) $\left|\left(v_{a}, \bar{D} \rho\left(f\left(z_{a}\right)\right)\right)\right|<\left(\varepsilon_{1}\right)^{2}$;

if $\left|z_{a}-z_{b}\right|<l(r)$ then:

(ii) if $\pi(a) \neq \pi(b):\left|\left(v_{a}, v_{b}\right)\right|<\left(\varepsilon_{1}\right)^{2}$,

(iii) if $\pi(a)=\pi(b):\left|v_{a}-v_{b}\right|,\left.\left|\left(v_{a}, v_{b}\right)-\right| v_{a}\right|^{2} \mid<\left(\varepsilon_{1}\right)^{2}$.

3.9 Define now for $z \in \bar{D}: h_{1}(z)=\sum_{a \in L} q_{a}(z) \cdot v_{a}$. This is the first stage correction function. The properties of the system of peak functions, and the choice of parameters above, including the choice of $v_{a}$ (that was made possible by Lemma 4), give to $h_{1}$ the following desirable properties.

3.10 Take $z \in W^{\prime}$; then the following hold:

(A) if $d\left(z, U_{1}\right) \geq r^{1 / 2}$, then $\left|h_{1}(z)\right|<\varepsilon_{1}$,

(B) $\left|\left(h_{1}(z), \bar{D} \rho(f(z))\right)\right|<\varepsilon_{1}$,

(C) $\left|h_{1}(z)\right|<\left(2 \varepsilon_{0}\right)^{-1} t(z) \kappa(z)+\varepsilon_{1}$,

(D) $\left|h_{1}(z)\right|>4 \varepsilon_{0} t(z) \kappa(z)-\varepsilon_{1}$.

Proof of (A) Assume first $d(z, b D) \geq r^{1 / 2}$, then by the definition of $\kappa=$ $\kappa_{r}:\left|h_{1}(z)\right| \leq \sum_{a \in L}\left|q_{a}(z)\right| \cdot\left|v_{a}\right| \leq \gamma_{0} \sum_{a \in L}\left|q_{a}(z)\right| \leq$ (by 3.3(i)) $\leq \gamma_{0} \cdot\left(\varepsilon_{0}\right)^{-1}$. $\kappa(z)+\left(\varepsilon_{1}\right)^{2}<\varepsilon_{1}$. Now when $d\left(z, U_{1}\right) \geq r^{1 / 2}$ and $d(z, b D)<r^{1 / 2}$ then $z^{*} \in b D \backslash U_{1}$ which implies $t(z)=0$ and $(\overline{\mathrm{A}})$, in this case, follows from (C).

\section{Proof of (B)}

$$
\begin{aligned}
\left|\left(h_{1}(z), \bar{D} \rho(f(z))\right)\right| \leq \sum_{a \in L}\left|q_{a}(z)\right| \cdot\left|\left(v_{a}, \bar{D} \rho(f(z))\right)\right| \\
=\sum_{\left(a \in L,\left|z_{a}-z\right| \leq r^{0.2}\right)}\left|q_{a}(z)\right| \cdot\left(\left|\left(v_{a}, \bar{D} \rho\left(f\left(z_{a}\right)\right)\right)\right|+\left|\bar{D} \rho(f(z))-\bar{D} \rho\left(f\left(z_{a}\right)\right)\right|\right) \\
\quad+\sum_{\left(a \in L,\left|z_{a}-z\right|>r^{0.2}\right)}\left|q_{a}(z)\right| \cdot\left|v_{a}\right| \cdot|\bar{D} \rho(f(z))| \quad(\text { by 3.8(i) and 3.5(i)) } \\
\quad<2\left(\varepsilon_{1}\right)^{2} \cdot \sum_{\left(a \in L,\left|z_{a}-z\right| \leq r^{0.2}\right)}\left|q_{a}(z)\right|+|\bar{D} \rho(f(z))| \cdot \gamma_{0} \cdot \sum_{\left(a \in L,\left|z_{a}-z\right|>r^{0.2}\right)}\left|q_{a}(z)\right|
\end{aligned}
$$

(in view of the definition of $\varepsilon_{1}$ in 3.5)

$$
<2\left(\varepsilon_{1}\right)^{2} \cdot \sum_{\left(a \in L,\left|z_{a}-z\right| \leq r^{0.2}\right)}\left|q_{a}(z)\right|+\left(\varepsilon_{1}\right)^{-0.1} \cdot \gamma_{0} \cdot \sum_{\left(a \in L,\left|z_{a}-z\right|>r^{0.2}\right)}\left|q_{a}(z)\right|
$$

(by 3.3(iii)) 
Proof of $(\mathrm{C})$

$$
\begin{aligned}
\left|h_{1}(z)\right| \leq & \sum_{a \in L}\left|q_{a}(z)\right| \cdot\left|v_{a}\right| \\
\leq & t(z) \cdot \sum_{\left(a \in L,\left|z_{a}-z\right| \leq r^{0.2}\right)}\left|q_{a}(z)\right|+\sum_{\left(a \in L,\left|z_{a}-z\right| \leq r^{0.2}\right)}\left|q_{a}(z)\right| \cdot\left|t(z)-t\left(z_{a}\right)\right| \\
& +\gamma_{0} \sum_{\left(a \in L,\left|z_{a}-z\right|>r^{0.2}\right)}\left|q_{a}(z)\right| \quad(\text { by 3.7, and 3.3(i), (iv)) } \\
< & \left(2 \varepsilon_{0}\right)^{-1} t(z) \kappa(z)+\varepsilon_{1} .
\end{aligned}
$$

Proof of (D) (1) Let $1 \leq i \leq N$, and define: $h_{1 i}(z)=\sum_{a \in L, \pi(a)=i} q_{a}(z) \cdot v_{a}$; then $h_{1}=h_{11}+\cdots+h_{1 N}$. In a similar way to (B) (or to [D1,6.28]) it can be proved that for all $1 \leq i, j \leq N, z \in W^{\prime}, i \neq j:\left|\left(h_{1 i}(z), h_{1 j}(z)\right)\right|<\left(\varepsilon_{1}\right)^{1.5}$.

(2) Let $i=i(z)$ (see 3.2(iii)); then by 3.3, 3.7, 3.8:

$$
\begin{aligned}
\left|h_{1 i}(z)\right| & >\kappa(z) \cdot\left|\sum_{a \in L_{i}} q_{a}\left(z^{*}\right) \cdot v_{a}\right|-\left(\varepsilon_{1}\right)^{2} \\
& >\kappa(z) \cdot\left|\sum_{\left(a \in L_{i},\left|z_{a}-z\right| \leq r^{0.2}\right)} q_{a}\left(z^{*}\right) \cdot v_{a}\right|-2\left(\varepsilon_{1}\right)^{2} \\
& >\kappa(z) \cdot t(z) \cdot\left|\sum_{\left(a \in L_{i},\left|z_{a}-z\right| \leq r^{0.2}\right)} q_{a}\left(z^{*}\right)\right|-\left(\varepsilon_{1}\right)^{1.5} \\
& >4 \cdot \kappa(z) \cdot t(z) \cdot \varepsilon_{0}-\left(\varepsilon_{1}\right)^{1.5} .
\end{aligned}
$$

Now from (1) and (2), (D) follows.

3.11 Define now for $z \in \bar{D}$ :

$$
h_{2}(z)=-(1 / 2) \sum_{a, b \in L} q_{a}(z) q_{b}(z) \cdot P_{\rho, f\left(z_{a}\right)}\left(v_{a}, v_{b}\right) \bar{D} \rho\left(f\left(z_{a}\right)\right) /\left|\bar{D} \rho\left(f\left(z_{a}\right)\right)\right|^{2},
$$

and define $h=h_{1}+h_{2}$. The function $h$ is almost equal to the final correction function $g$ in $W^{\prime}$. By the proof of (B) below $h_{2}$ is well defined.

3.12 Take $z \in W^{\prime}$; then the following hold for all $z \in W^{\prime}$ :
(A) if $d\left(z, U_{1}\right) \geq r^{1 / 2}$, then $\left|h_{2}(z)\right|<2 \varepsilon_{1}$,
(B) $\left|h_{2}(z)\right|<\gamma_{0}^{1 / 2}\left|h_{1}(z)\right|+\varepsilon_{1}$,
(C) $\left|2\left(h_{2}(z), \bar{D} \rho(f(z))\right)+P_{\rho, f(z)}\left(h_{1}(z)\right)\right|<\varepsilon_{1}$,
(D) $\left|\left(h_{1}(z), h_{2}(z)\right)\right|<\varepsilon_{1}$,
(E) $|h(z)|<\left(\gamma_{0}\right)^{1 / 2}$.

Proof. The proof of (A) follows from (A) of 3.10 and (B) here. The proof of (D) is a simple consequence of $3.8(\mathrm{i})$ and 3.3 and is similar to the proof of 3.10(B). Now (E) follows from (B) and 3.10(C) and 3.4. It remains to show (B) and $(\mathrm{C})$.

Proof of (B) Note first that by 3.7: $P_{\rho, f\left(z_{a}\right)}\left(v_{a}, v_{b}\right)=0$ whenever $a \in L$ and $z_{a} \in b D \backslash U_{1}$ or $z_{b} \in b D \backslash U_{1}$. Hence, in the following calculation only $a, b \in L$ where $z_{a}, z_{b} \in U_{1}$ should be considered. Recall also that when $a, b \in L$ and 
$z_{a} \in U_{1}$ then by $3.7 f\left(z_{a}\right) \in K_{2}$. Now by 3.4: $\left|P_{\rho, f\left(z_{a}\right)}\left(v_{a}, v_{b}\right)\right|<C t_{a} t_{b}$ and $1 /\left|\bar{D} \rho\left(f\left(z_{a}\right)\right)\right|<C$. Therefore:

$$
\begin{aligned}
\left|h_{2}(z)\right| & \leq \sum_{a, b \in L}\left|q_{a}(z) q_{b}(z)\right| \cdot C \cdot t_{a} t_{b} /\left|\bar{D} \rho\left(f\left(z_{a}\right)\right)\right| \\
\leq & \sum_{\left(a, b \in L,\left|z_{a}-z\right|+\left|z_{b}-z\right| \leq 2 r^{0.2}\right)}\left|q_{a}(z) q_{b}(z)\right| \cdot C^{2} \cdot \gamma_{0}\left(t(z)+\varepsilon_{1}\right) \\
& +\sum_{\left(a, b \in L,\left|z_{a}-z\right|+\left|z_{b}-z\right| \geq 2 r^{0.2}\right)}\left|q_{a}(z) q_{b}(z)\right| \cdot C^{2} \cdot \gamma_{0}^{2} \\
\leq & \left(\sum_{a \in L}\left|q_{a}(z)\right|\right)^{2} \gamma_{0}^{3 / 4} t(z)+\left(\sum_{\left(b \in L,\left|z_{b}-z\right| \geq r^{0.2}\right)}\left|q_{a}(z)\right|\right)\left(\sum_{a \in L}\left|q_{a}(z)\right|\right)+\varepsilon_{1} / 2 \\
\leq & \left(\varepsilon_{0}\right)^{-2} \kappa(z)^{2} \gamma_{0}^{3 / 4} t(z)+\varepsilon_{1}<\gamma_{0}^{1 / 2}\left|h_{1}(z)\right|+\varepsilon_{1} .
\end{aligned}
$$

The size of $\gamma_{0}>0$ (see 3.4) with 3.3 was used in the last four inequalities. The last inequality also uses $3.10(\mathrm{D})$ and the fact that $0<\kappa(z) \leq 1$.

Proof of (C)

$$
\begin{aligned}
\left|2\left(h_{2}(z), \bar{D} \rho(f(z))\right)+P_{f(z)}\left(h_{1}(z)\right)\right| \\
=\mid \sum_{a, b \in L} q_{a}(z) q_{b}(z) \cdot P_{f(z)}\left(v_{a}, v_{b}\right) \\
\quad-\sum_{a, b \in L} q_{a}(z) q_{b}(z) \cdot P_{f\left(z_{a}\right)}\left(v_{a}, v_{b}\right)\left(\bar{D} \rho\left(f\left(z_{a}\right)\right) /\left|\bar{D} \rho\left(f\left(z_{a}\right)\right)\right|^{2}, \bar{D} \rho(f(z))\right) \mid \\
\quad \text { (applying the considerations mentioned in the proof of (B) above) } \\
\leq \quad \sum_{\left(a, b \in L,\left|z_{a}-z\right|+\left|z_{b}-z\right| \leq 2 r^{0.2}\right)}\left|q_{a}(z) q_{b}(z)\right| \\
\quad \cdot\left|P_{f(z)}\left(v_{a}, v_{b}\right)-P_{f\left(z_{a}\right)}\left(v_{a}, v_{b}\right) \cdot\left(\bar{D} \rho\left(f\left(z_{a}\right)\right) /\left|\bar{D} \rho\left(f\left(z_{a}\right)\right)\right|^{2}, \bar{D} \rho(f(z))\right)\right| \\
\quad+|\bar{D} \rho(f(z))| \cdot \sum_{\left(a, b \in L,\left|z_{a}-z\right|+\left|z_{b}-z\right|>2 r^{0.2}\right)}\left|q_{a}(z) \bar{q}_{b}(z)\right| \cdot C^{2} t_{a} t_{b}
\end{aligned}
$$

(consider the size of $\gamma_{0}>0$ and $\varepsilon_{0}>0$ in 3.4, 3.5 and apply 3.3)

$$
\begin{aligned}
\leq & \sum_{a, b \in L}\left|q_{a}(z) q_{b}(z)\right| \cdot\left(\varepsilon_{1}\right)^{1.5} \\
& +\left(\sum_{a \in L,\left|z_{a}-z\right|>r^{0.2}}\left|q_{a}(z)\right|\right)\left(\sum_{a \in L}\left|q_{a}(z)\right|\right) \cdot \gamma_{0}<\varepsilon_{1} .
\end{aligned}
$$

3.13 We can now estimate $\rho(f(z)+h(z))-\rho(f(z))$ in $W^{\prime}$. Note that since by 3.12(E) for $z \in W^{\prime}:|h(z)|<\delta_{0} / 2$, then if $d\left(z, U_{1}\right)<r^{1 / 2}$ by 3.7: $f(z) \in K_{2}$ and by 3.4: $\left|\alpha_{f(z)}(h(z))\right|<|h(z)|^{2}$.

3.14 For all $z \in W^{\prime}$ :

$$
C|h(z)|^{2}+\varepsilon^{4}>\rho(f(z)+h(z))-\rho(f(z))>|h(z)|^{2}-\varepsilon^{4} .
$$

Proof. (1) Assume first that $d\left(z, U_{1}\right) \geq r^{1 / 2}$; then by 3.10(A) and 3.12(A): 
$|h(z)|<3 \varepsilon_{1}$ and therefore by 3.5: $|\rho(f(z)+h(z))-\rho(f(z))|<\varepsilon^{10}$. It is clear that in this case 3.14 holds.

(2) Henceforth we will assume $d\left(z, U_{1}\right)<r^{1 / 2}$. By 1.3, 1.5:

$$
\begin{aligned}
& \rho(f(z)+h(z))-\rho(f(z)) \\
&= \operatorname{Re}\left(2(h(z), \bar{D} \rho(f(z)))+\left(P_{f(z)}\right)^{2}(h(z))\right)+H_{f(z)}(h(z))+\alpha_{f(z)}(h(z)) \\
& \quad(\text { applying } 3.13 \text { and the assumption that } \rho \text { is normalized-3) } \\
& \geq \operatorname{Re}\left(2(h(z), \bar{D} \rho(f(z)))+\left(P_{f(z)}\right)^{2}(h(z))\right)+3|h(z)|^{2}-|h(z)|^{2} \\
&= \operatorname{Re}\left(2\left(h_{2}(z), \bar{D} \rho(f(z))\right)+\left(P_{f(z)}\right)^{2}\left(h_{1}(z)\right)\right)+2|h(z)|^{2} \\
&+\operatorname{Re}\left(2\left(h_{1}(z), \bar{D} \rho(f(z))\right)+2 P_{f(z)}\left(h_{1}(z), h_{2}(z)\right)+\left(P_{f(z)}\right)^{2}\left(h_{2}(z)\right)\right) \\
& \quad(\text { by } 3.10(\mathrm{~B}) \text { and } 3.12(\mathrm{C})) \\
&> 2|h(z)|^{2}+\operatorname{Re}\left(2 P_{f(z)}\left(h_{1}(z), h_{2}(z)\right)+\left(P_{f(z)}\right)^{2}\left(h_{2}(z)\right)\right)-3 \varepsilon_{1} \\
& \quad\left(\text { by the fact that } f(z) \in K_{2} \text { and by } 3.4\right) \\
& \geq 2|h(z)|^{2}-2 C\left|h_{1}(z)\right|\left|h_{2}(z)\right|-C\left|h_{2}(z)\right|^{2}-3 \varepsilon_{1} \quad(\text { by } 3.12(\mathrm{~B})) \\
& \geq 2|h(z)|^{2}-2 C\left(\gamma_{0}\right)^{1 / 2}\left|h_{1}(z)\right|^{2}-C \gamma_{0}\left|h_{1}(z)\right|^{2}-4 \varepsilon_{1} \quad(\text { by } 3.4) \\
& \geq|h(z)|^{2}-4 \varepsilon_{1} .
\end{aligned}
$$

(by $3.10(B)$ and $3.12(C)$ )

(3) In view of 3.13 and the calculations above we have:

$$
\begin{aligned}
\rho(f(z) & +h(z))-\rho(f(z)) \\
\leq & \operatorname{Re}\left(2(h(z), \bar{D} \rho(f(z)))+\left(P_{f(z)}\right)^{2}(h(z))\right)+2 H_{f(z)}(h(z)) \\
\leq & \operatorname{Re}\left(2\left(h_{2}(z), \bar{D} \rho(f(z))\right)+\left(P_{f(z)}\right)^{2}\left(h_{1}(z)\right)\right)+(C / 2)|h(z)|^{2} \\
& +\operatorname{Re}\left(2\left(h_{1}(z), \bar{D} \rho(f(z))\right)+2 P_{f(z)}\left(h_{1}(z), h_{2}(z)\right)+\left(P_{f(z)}\right)^{2}\left(h_{2}(z)\right)\right) \\
& \quad(\text { by }(3.10)(\mathrm{B}), 3.12(\mathrm{C}) \text { and 3.4) } \\
\leq & (C / 2)|h(z)|^{2}+2 C\left|h_{1}(z)\right| \cdot\left|h_{2}(z)\right|+C\left|h_{2}(z)\right|^{2}+2 \varepsilon_{1}
\end{aligned}
$$$$
\text { (by } 3.12(\mathrm{~B}) \text { ) }
$$$$
\leq C|h(z)|^{2}+3 \varepsilon_{1}
$$

3.15 By now we have done the work needed to show that the function $h$ fulfills the requirements of $g$ in $W^{\prime}$. We will correct $h$ slightly in $W^{\prime}$, to make it very small in $\bar{D} \backslash W^{\prime}$. Then the lemma will hold in all $\bar{D}$. Applying the globalization process of $[D 1,1.34-1.38]$, we obtain that there exists a $C^{\infty}$ map, $g_{1}: \bar{D} \rightarrow \mathbb{C}^{M}$, which is holomorphic in $D$ such that:

(i) for all $z \in W^{\prime}:\left|g_{1}(z)-h(z) / S(z)\right|<\left(\varepsilon_{1}\right)^{1 / 2}$,

(ii) for all $z \in \bar{D} \backslash W^{\prime}:\left|g_{1}(z)\right|<\left(\varepsilon_{1}\right)^{1 / 2}$.

Define for $z \in \bar{D}: g(z)=g_{1}(z) \cdot S(z)$. As commented in 3.3, when $D=B^{N}$ the globalization process is not needed and in this case we take: $g=h$.

3.16 We are now ready to show that each of the items in Lemma 1 is proved. Clearly 3.15 is part of every proof and we will avoid repeating it for each of the items.

Let $z \in \bar{D}$. If $d\left(z, U_{1}\right) \geq r^{1 / 2}$ then by $3.10(\mathrm{~A})$ and $3.12(\mathrm{~A})|g(z)|<\left(\varepsilon_{1}\right)^{1 / 3}$ and therefore by $3.5|g(z)|<d(f(z), b \Omega)$. Now if $d\left(z, U_{1}\right)<r^{1 / 2}$ then by $3.7 f(z) \in K_{2}$ and by $3.12(\mathrm{E})|g(z)|<2\left(\gamma_{0}\right)^{1 / 2}<$ (by 3.4$)<d\left(K_{2}, b \Omega\right) \leq$ $d(f(z), b \Omega)$. In both cases $f(z)+g(z) \in \Omega$ and hence (a) follows. 
Now (b) follows from 3.14; (c) from 3.7, 3.10(D) and 3.12(D). Item (d) in Lemma 1 comes from $3.10(\mathrm{C})$ and $3.12(\mathrm{~B})$ and 3.7, and $(\mathrm{e})$ is a consequence of 3.10(A), 3.12(A) and the fact that (by 3.4, 3.5) $r^{1 / 2}<d(K, b D)$. Finally since $D^{\alpha} S(P)=0$ when $\alpha \in \mathbf{N}^{N}$ and $|\alpha| \leq k$, then by 3.15 this is also true for $g$. In the case that $D=B^{N}$ (f) follows from 3.3.

\section{Proof of LemMa 2}

The correction function $h$ that we are about to construct is a sum of two stage correction functions: $h=h_{1}+h_{2}$. The construction of $h_{1}$ depends on Lemma 4 and requires only one codimension. In the construction of $h_{2}$ Lemma 7 is applied and therefore we must assume that $M \geq \max \{2 N, N+3\}$. In the case that $w_{0}$ is a point of strict convexity (as defined in 1.5) with respect to $\rho$, then the construction of $h_{2}$ is not needed and we can take $h=h_{1}$. We would then follow the rest of the proof with very slight modifications. Thus in this case only one codimension is needed $(M \geq N+1)$.

In a more general situation, when $w_{0}$ is a strict local minimum point (which is not necessarily nondegenerate), the result of Lemma 10 is accomplished by a variation on the proof of Lemma 10 above, where Lemma 1 is applied repeatedly with use of the minimality of $w_{0}$ to maintain a positive lower bound to $d\left(f_{n}(b D), w_{0}\right)$ during the process. We will not elaborate on the details. 1.

Let $\alpha_{w}=\alpha_{\rho, w}, P_{w}=P_{\rho, w}, H_{w}=H_{\rho, w}$ be as in Definition 1.3 and Lemma

4.1 Fix $1>\delta>0$ such that $\bar{B}\left(w_{0}, 4 \delta\right) \subset \Omega$ and when $w \in \bar{B}\left(w_{0}, 4 \delta\right)$ then:

(i) $|\bar{D} \rho(w)|<\varepsilon_{0}$,

(ii) for all $1 \leq i, j \leq M:\left|D_{i} D_{j} \rho(f(w))-D_{i} D_{j} \rho\left(f\left(w_{0}\right)\right)\right|<\varepsilon_{0} / M^{2}$.

We obtain that for all $v, u \in b B^{M}$ :

(iii) $\left|P_{w}(v, u)-P_{w_{0}}(v, u)\right|<\varepsilon_{0}$.

Define $G=B\left(w_{0}, \delta\right), G^{\prime}=B\left(w_{0}, 2 \delta\right)$ and $G^{\prime \prime}=B\left(w_{0}, 4 \delta\right)$. For brevity's sake we will denote $\mathbf{P}=P_{\rho}, w_{0}$.

4.2 We will use some of the terms defined in the proof of Lemma 1. Let $L, L_{i},\left\{z_{a}: a \in L\right\}, W, W^{\prime}, W^{*}, U, U^{*},\left\{q_{a}: a \in L\right\}, z \rightarrow z^{*}$ be as in the proof of Lemma 1,3.1-3.5, where the size of $r>0$ in the definitions of $L,\left\{z_{a}: a \in L\right\}$ and $\left\{q_{a}: a \in L\right\}$ is bounded from above in 4.5 and 4.10 below.

4.3 Take $\delta_{0}>0$, such that $\delta>\delta_{0}$ and for all $w \in \bar{G}^{\prime}$ and $v \in \mathbb{C}^{M},|v|<$ $\delta_{0}:\left|\alpha_{w}(v)\right|<(1 / 2)|v|^{2}$. Define: $C=\max \left\{|\mathbf{P}(v, u)|: v, u \in b B^{M}\right\}, C^{\prime}=$ $\max \{|S(z)|: z \in \bar{D}\}$ and put $\alpha_{0}=\left(\varepsilon_{0} \delta_{0} /(C+1)\right)^{100}$. Now let $\alpha_{0} \geq \alpha>0$ and assume (as we may) that $0<\varepsilon<\left(\alpha /\left(1+1 / d(f(\bar{D}), b \Omega)+C^{\prime}\right)\right)^{100}$.

4.4 Take $0<\varepsilon_{1}$ where $\varepsilon_{1}<\varepsilon^{10} \cdot(1+\max \{|\bar{D} \rho(w)|: w \in f(\bar{D})\})^{-10}$, and for all $w \in f(\bar{D}) \cup \bar{G}^{\prime \prime}, v \in\left(\varepsilon_{1}\right)^{0.1} \cdot B^{M}:|\rho(w+v)-\rho(w)|<\varepsilon^{10}$.

4.5 Assume that $r>0$ is small enough so that $r<\varepsilon_{1}^{\left(1 / \varepsilon_{1}\right)}$ and when $z, w \in$ $\bar{D},|z-w|<l(r)$ then:

(i) $|f(z)-f(w)|<\left(\varepsilon_{1}\right)^{2}$,

(ii) $|\bar{D} \rho(f(z))-\bar{D} \rho(f(w))|<\left(\varepsilon_{1}\right)^{2}$,

(iii) for all $1 \leq i, j \leq M:\left|D_{i} D_{j} \rho(f(z))-D_{i} D_{j} \rho(f(w))\right|<\left(\varepsilon_{1}\right)^{2} / M^{2}$.

We obtain from (iii) that for all $v, u \in b B^{M}$ :

(iv) $\left|P_{f(z)}(v, u)-P_{f(w)}(v, u)\right|<\left(\varepsilon_{1}\right)^{2}$.

4.6 Assume that 3.2, 3.3 hold now with $\varepsilon_{1}>0$ chosen above (in 4.3-4.4) 
and $r>0$, whose choice is finalized in 4.10. We will continue to use the abbreviation: $l(r)=-1 / \log (r)$.

4.7 Define $U_{1}=\left\{z \in U^{*}: d\left(z, f^{-1}(G)\right) \leq l(r)\right\}$. Now take $z \in b D$ and let $t(z)=\alpha$ when $z \in U_{1}$ and $d\left(z, b U_{1}\right) \geq l(r)$. If $z \in U_{1}$ and $d\left(z, b U_{1}\right) \leq l(r)$ then put $t(z)=\max \left\{\alpha \cdot d\left(z, b U_{1}\right) / l(r), \varepsilon_{1}\right\}$. When $z \in b D \backslash U_{1}$ then let: $t(z)=\varepsilon_{1}$. Define it now on $W^{\prime}$, put for $z \in W^{\prime}: t(z)=t\left(z^{*}\right)$. The function $t$ is continuous on $W^{\prime} \cup b D$. For $a \in L$ define $t_{a}=t\left(z_{a}\right)$.

4.8 By Lemma 7 there exist $C^{1}$ functions $u, v_{1}, \ldots, v_{N}: b D \rightarrow b B^{M}$ such that for all $z \in b D, 1 \leq i \leq N$ :

(1) $|(u(z), \bar{D} \rho(f(z)))-|(u(z), \bar{D} \rho(f(z)))||<\left(\varepsilon_{1}\right)^{2}$,

(2) $\mathbf{P}(u(z), u(z))=0$,

(3) $\left\{v_{1}(z), \ldots, v_{n}(z)\right\}$ is orthonormal,

(4) $\left|\left(v_{i}(z), \bar{D} \rho(f(z))\right)\right|<\left(\varepsilon_{1}\right)^{2}$,

(5) $\left|\left(v_{i}(z), u(z)\right)\right|<\left(\varepsilon_{1}\right)^{2}$,

(6) $\left|\mathbf{P}\left(v_{i}(z), u(z)\right)\right|=\left|\left(v_{i}(z), \mathbf{P}^{*} \bar{u}(z)\right)\right|<\left(\varepsilon_{1}\right)^{2}$.

Extend these functions continuously to $b D \cup W^{\prime}$ by defining for $z \in W^{\prime}$ and $1 \leq i \leq N: v_{i}(z)=v_{i}\left(z^{*}\right), u(z)=u\left(z^{*}\right)$.

4.9 Define for $z \in W^{\prime}$ :

$$
\mu(z)=\min \left\{t(z)^{-1.5},(2|(u(z), \bar{D} \rho(f(z)))|)^{-1}\right\} \quad \text { and } \quad s(z)=t(z)(\mu(z))^{1 / 2} ;
$$

then $s, \mu: W^{\prime} \rightarrow \mathbb{R}^{+}$are continuous and $0<s(z) \leq t(z)^{1 / 4}$.

4.10 Assume now that $r>0$ was chosen small enough so that when $z, w \in$ $W^{\prime}$, where $|z-w|<l(r)$, and $1 \leq i \leq N$ then:

$\left|v_{i}(z)-v_{i}(w)\right|,|u(z)-u(w)|,|t(z)-t(w)|,|\mu(z)-\mu(w)|,|s(z)-s(w)|<\left(\varepsilon_{1}\right)^{2}$.

Define then for $1 \leq i \leq N, a \in L_{i}: v_{a}=v_{i}\left(z_{a}\right), u_{a}=u\left(z_{a}\right), \mu_{a}=\mu\left(z_{a}\right), s_{a}=$ $s\left(z_{a}\right)$.

4.11 Define for $z \in \bar{D}: h_{1}(z)=\sum_{a \in L} q_{a}(z) t_{a} v_{a}$.

4.12 Take $z \in W^{\prime}$; then the following hold:

(A) if $d\left(z, U_{1}\right) \geq l(r)$ then $\left|h_{1}(z)\right|<\varepsilon_{1}$,

(B) $\left|\left(h_{1}(z), \bar{D} \rho(f(z))\right)\right|<\varepsilon_{1}$,

(C) $\left|\left(h_{1}(z), u(z)\right)\right|<\varepsilon_{1}$,

(D) $\left|\left(h_{1}(z), \mathbf{P}^{*} \bar{u}(z)\right)\right|<\varepsilon_{1}$,

(E) $\left|h_{1}(z)\right|<\left(\varepsilon_{0}\right)^{-1} t(z) \kappa(z)+\varepsilon_{1}$,

(F) $\left|h_{1}(z)\right|>2 \varepsilon_{0} t(z) \kappa(z)-\varepsilon_{1}$,

(G) when $z \in W^{\prime}$ and $f(z) \notin G^{\prime}$ then: $\left|h_{1}(z)\right|<\varepsilon_{1}$.

Proof. The proofs of (A)-(F) contain the same calculations as the proofs of (A) $-(D)$ in 3.10. The proofs of (C) and (D) are derived from 4.8(5), 4.8(6), and (B) is derived from 4.8(4) and they are all very similar to the proof of 3.10(B).

To prove (G) we would show that when $z \in W^{\prime}$ and $f(z) \notin G^{\prime}$ then $d\left(z, U_{1}\right) \geq l(r)$ and then (A) can be applied. Note that if $z \in W^{\prime}$ and $d\left(z, U_{1}\right)<l(r)$ then by 4.7 there exists $z^{\prime} \in f^{-1}(G)$ so that $\left|z-z^{\prime}\right|<2 l(r)$. Thus by $4.5\left|f(z)-f\left(z^{\prime}\right)\right|<\varepsilon_{1}$; now since $\left|f\left(z^{\prime}\right)-w_{0}\right|<\delta$ and (by 4.3, 4.4) $\varepsilon_{1}<\varepsilon<\alpha<\delta_{0}<\delta$ then $\left|f(z)-w_{0}\right|<2 \delta$ and $f(z) \in G^{\prime}$.

4.13 Define now for $z \in \bar{D}$ :

$$
h_{2}(z)=-\sum_{a, b \in L} q_{a}(z) q_{b}(z) \cdot \mathbf{P}\left(v_{a}, v_{b}\right) \cdot s_{a} \cdot s_{b} \cdot u_{a},
$$


and $h=h_{1}+h_{2}$. The role of $h_{2}$ is clearly illustrated by (C) below and 4.8. They imply that in the estimate of

$$
\begin{aligned}
\rho(f(z)+h(z))-\rho(f(z))= & \operatorname{Re}\left(2(h(z), \bar{D} \rho(f(z)))+\left(P_{f(z)}\right)^{2}(h(z))\right) \\
& +H_{f(z)}(h(z))+\alpha_{f(z)}(h(z)),
\end{aligned}
$$

in the set $f^{-1}\left(G^{\prime}\right)$, the function $h_{2}$ will be neutral toward $\mathbf{P}$ (which is very close to $\left.P_{f(z)}\right)$ in the sense of (D), (F) below. At the same time $h_{2}$ is almost perpendicular to $h_{1}$ and therefore it contributes to the positive Hessian $H_{f(z)}(h(z))$. This contribution is significant since by $(\mathrm{C}) h_{2}$ is very large in comparison with the term: $\mathbf{P}\left(h_{1}(z), h_{1}(z)\right)$.

4.14 Take $z \in W^{\prime}$; then the following hold:

(A) if $d\left(z, U_{1}\right) \geq l(r)$ then $\left|h_{2}(z)\right|<\varepsilon_{1}$,

(B) $\left|h_{2}(z)\right|<\left(\varepsilon_{0}\right)^{-2} \cdot C \cdot t(z)^{1 / 2} \kappa(z)^{2}+\varepsilon_{1}$,

(C) define $\xi(z)=h_{2}(z)+\mathbf{P}\left(h_{1}(z), h_{1}(z)\right) \cdot \mu(z) \cdot u(z)$,

then: $|\xi(z)|<\varepsilon_{1}$ (recall that $\mu(z)$ is positive and $u(z) \in \mathbb{C}^{M}$ )

(D) $\left|\mathbf{P}\left(h_{2}(z), h_{2}(z)\right)\right|<\varepsilon_{1}$,

(E) $\left|\left(h_{1}(z), h_{2}(z)\right)\right|<\varepsilon_{1}$,

(F) $\left|\mathbf{P}\left(h_{1}(z), h_{2}(z)\right)\right|<\varepsilon_{1}$,

(G) $|h(z)|<\alpha^{1 / 3} \cdot \kappa_{r}(z)^{2}+\varepsilon_{1}<\delta_{0} / 2$,

(H) when $z \in W^{\prime}$ and $f(z) \notin G^{\prime}$ then: $\left|h_{2}(z)\right|<\varepsilon_{1}$.

Proof. The proofs of (A) and (B) are routine applications of calculations from 3.10, 3.12. Now (G) follows from 4.12(E) and 4.14(B) and the definition of $t(z)$ in 4.7 (note that $\alpha \geq t(z)$ ) and the size of $\alpha>0$ in 4.3. The proof of $(\mathrm{H})$ is the same as $4.12(\mathrm{G})$. We begin then with the proof of $(\mathrm{C})$ that will later imply (D)-(F).

Proof of $(\mathrm{C})$.

$$
\begin{aligned}
\mid h_{2}(z) & +\mathbf{P}\left(h_{1}(z), h_{1}(z)\right) \cdot \mu(z) \cdot u(z) \mid \\
= & \left|\sum_{a, b \in L} q_{a}(z) q_{b}(z) \cdot \mathbf{P}\left(v_{a}, v_{b}\right) t_{a} t_{b}\left(\mu(z) u(z)-\left(\mu_{a} \mu_{b}\right)^{1 / 2} u_{a}\right)\right| \\
\leq & \sum_{\left(a, b \in L,\left|z_{a}-z\right|+\left|z_{b}-z\right| \leq 2 r^{0.2}\right)}\left|q_{a}(z) q_{b}(z)\right| C \alpha^{2}\left|\mu(z) u(z)-\left(\mu_{a} \mu_{b}\right)^{1 / 2} u_{a}\right| \\
& +\sum_{\left(a, b \in L,\left|z_{a}-z\right|+\left|z_{b}-z\right|>2 r^{0.2}\right)}\left|q_{a}(z) q_{b}(z)\right| 2 C \alpha^{2}\left(\varepsilon_{1}\right)^{-1.5} \\
\leq & \left|\sum_{\left(a, b \in L,\left|z_{a}-z\right|+\left|z_{b}-z\right| \leq 2 r^{0.2}\right)}(z) q_{b}(z)\right| \cdot\left(\varepsilon_{1}\right)^{2} \\
& +\left(\sum_{a \in L,\left|z_{a}-z\right|>r^{0.2}}\left|q_{a}(z)\right|\right) \cdot\left(\sum_{b \in L}\left|q_{b}(z)\right|\right) \cdot \alpha \cdot\left(\varepsilon_{1}\right)^{-1.5}
\end{aligned}
$$

(by $3.3(\mathrm{iii}))<\varepsilon_{1}$.

Proof of (D).

$$
\begin{aligned}
\left|\mathbf{P}^{2}\left(h_{2}(z)\right)\right| & =\left|\mathbf{P}^{2}\left(\mathbf{P}^{2}\left(h_{1}(z)\right) \cdot \mu(z) \cdot u(z)-\xi(z)\right)\right| \\
& \leq\left|\left(\mathbf{P}^{2}\left(h_{1}(z)\right) \cdot \mu(z)\right)^{2} \cdot \mathbf{P}^{2}(u(z))\right|+\varepsilon_{1}=(\text { by } 4.8(2))=\varepsilon_{1} .
\end{aligned}
$$


Proof of $(\mathrm{E})$.

$$
\begin{aligned}
& \left|\left(h_{1}(z), h_{2}(z)\right)\right| \\
& \quad<\left|\left(h_{1}(z), \mathbf{P}^{2}\left(h_{1}(z)\right) \cdot \mu(z) \cdot u(z)\right)\right|+\varepsilon_{1} / 2 \\
& \quad \leq(1 / 2)\left|\left(h_{1}(z), u(z)\right)\right|+\varepsilon_{1} / 2<(\text { apply now } 4.12(\mathrm{C}))<\varepsilon_{1} .
\end{aligned}
$$

Proof of $(\mathrm{F})$.

$$
\begin{gathered}
\left|\mathbf{P}\left(h_{1}(z), h_{2}(z)\right)\right|<\left|\mathbf{P}\left(h_{1}(z), \mathbf{P}^{2}\left(h_{1}(z)\right) \cdot \mu(z) \cdot u(z)\right)\right|+\varepsilon_{1} / 2 \\
\leq\left|\left(h_{1}(z), \mathbf{P}^{*} \bar{u}(z)\right)\right| / 2+\varepsilon_{1} / 2<(\text { apply } 4.12(\mathrm{D}))<\varepsilon_{1} .
\end{gathered}
$$

\subsection{Proposition. For all $z \in W^{\prime}$ :}

$$
\rho(f(z)+h(z))-\rho(f(z))>\left|h_{1}(z)\right|^{2} \cdot \min \left\{1,\left(\alpha^{-1 / 2} \kappa(z)^{2}-\alpha^{-1 / 4}\right)\right\}-\varepsilon^{2} .
$$

Proof. First note that if $f(z) \notin G^{\prime}$ then by $4.12(\mathrm{G}), 4.14(\mathrm{H}),|h(z)|,\left|h_{1}(z)\right|<$ $2 \varepsilon_{1}$ and thus (see 4.4) $|\rho(f(z)+h(z))-\rho(f(z))|<\varepsilon^{5}$. This implies the proposition when $f(z) \notin G^{\prime}$. Therefore we will assume from now on that $f(z) \in G^{\prime}$. Now by $4.14(\mathrm{G})$, for all $z \in W^{\prime},|h(z)|<\delta_{0}$. This gives us by 4.3: $\left|\alpha_{f(z)}(h(z))\right|<|h(z)|^{2} / 2$. By the assumption that $\rho$ is normalized-3 (see Definitions 1.3, 1.5), we get:

$$
\begin{aligned}
& \rho(f(z)+h(z))-\rho(f(z)) \\
& \quad=\operatorname{Re}\left(2(h(z), \bar{D} \rho(f(z)))+\left(P_{f(z)}\right)^{2}(h(z))\right)+H_{f(z)}(h(z))+\alpha_{f(z)}(h(z)) \\
& \geq \operatorname{Re}\left(2(h(z), \bar{D} \rho(f(z)))+\left(P_{f(z)}\right)^{2}(h(z))\right)+2.5|h(z)|^{2} .
\end{aligned}
$$

4.16 Using 4.12(B) we get from this:

$$
\begin{aligned}
& \rho(f(z)+h(z))-\rho(f(z)) \\
& \quad \geq \operatorname{Re}\left(2\left(h_{2}(z), \bar{D} \rho(f(z))\right)+\left(P_{f(z)}\right)^{2}(h(z))\right)+2.5|h(z)|^{2}-2 \varepsilon_{1} .
\end{aligned}
$$

4.17 Now by $4.1\left|\left(P_{f(z)}\right)^{2}(h(z))-\mathbf{P}^{2}(h(z))\right|<\varepsilon_{0} \cdot|h(z)|^{2}$; therefore 4.16 gives

$$
\begin{aligned}
& \rho(f(z)+h(z))-\rho(f(z)) \\
& \quad \geq \operatorname{Re}\left(2\left(h_{2}(z), \bar{D} \rho(f(z))\right)+\mathbf{P}^{2}(h(z))\right)+2|h(z)|^{2}-2 \varepsilon_{1} .
\end{aligned}
$$

4.18 Applying $4.14(\mathrm{D})$, (F) we get

$$
\begin{aligned}
\operatorname{Re} \mathbf{P}^{2}(h(z)) & =\operatorname{Re}\left(\mathbf{P}^{2}\left(h_{1}(z)\right)+\mathbf{P}^{2}\left(h_{2}(z)\right)+2 \mathbf{P}\left(h_{1}(z), h_{2}(z)\right)\right) \\
& \geq \operatorname{Re}\left(\mathbf{P}^{2}\left(h_{1}(z)\right)\right)-2 \varepsilon_{1} .
\end{aligned}
$$

Now applying first $4.14(C)$ and then $4.8(1)$ we get:

$$
\begin{aligned}
\operatorname{Re}\left(h_{2}(z), \bar{D} \rho(f(z))\right) & \geq-\operatorname{Re}\left(\mathbf{P}^{2}\left(h_{1}(z)\right) \cdot \mu(z) \cdot(u(z), \bar{D} \rho(f(z)))\right)-\varepsilon^{5} \\
& \geq-\operatorname{Re}\left(\mathbf{P}^{2}\left(h_{1}(z)\right) \cdot \mu(z) \cdot|(u(z), \bar{D} \rho(f(z)))|\right)-2 \varepsilon^{5} .
\end{aligned}
$$

4.19 We conclude from this and from 4.17 that:

$$
\begin{aligned}
\rho(f(z) & +h(z))-\rho(f(z)) \\
& \geq \operatorname{Re}\left(-2 \mathbf{P}^{2}\left(h_{1}(z)\right) \cdot \mu(z) \cdot|(u(z), \bar{D} \rho(f(z)))|+\mathbf{P}^{2}\left(h_{1}(z)\right)\right)+2|h(z)|^{2}-\varepsilon^{4} \\
& =\operatorname{Re}\left(\mathbf{P}^{2}\left(h_{1}(z)\right)(1-2 \mu(z) \cdot|(u(z), \bar{D} \rho(f(z)))|)\right)+2|h(z)|^{2}-\varepsilon^{4} .
\end{aligned}
$$

4.20 Recall that in 4.9 we defined:

$$
\mu(z)=\min \left\{t(z)^{-1.5}, 1 /(2 \cdot|(u(z), \bar{D} \rho(f(z)))|)\right\} .
$$


Now when $\mu(z)=(1 / 2)|(u(z), \bar{D} \rho(f(z)))|$, it is clear that Proposition 4.15 holds. Assume therefore that this is not the case. Then $\mu(z)=t(z)^{-1.5}$ and 4.19 yields: $\rho(f(z)+h(z))-\rho(f(z)) \geq-\left|\mathbf{P}^{2}\left(h_{1}(z)\right)\right|+2|h(z)|^{2}-\varepsilon^{4}$.

4.21 Looking at 4.14(E) we get that

$$
\rho(f(z)+h(z))-\rho(f(z)) \geq-\left|\mathbf{P}^{2}\left(h_{1}(z)\right)\right|+2\left|h_{1}(z)\right|^{2}+2\left|h_{2}(z)\right|^{2}-2 \varepsilon^{4} .
$$

In the case that $\left|\mathbf{P}^{2}\left(h_{1}(z)\right)\right|<\left|h_{1}(z)\right|^{2}$ the proof of Proposition 4.15 is concluded. So henceforth assume that $\left|\mathbf{P}^{2}\left(h_{1}(z)\right)\right| \geq\left|h_{1}(z)\right|^{2}$.

4.22 Thus by $4.14(C)$ :

$$
\begin{aligned}
\left|h_{2}(z)\right|^{2} & \geq\left|\mathbf{P}^{2}\left(h_{1}(z)\right)\right|^{2} \mu(z)^{2}-\varepsilon_{1} \\
& \geq\left|h_{1}(z)\right|^{4} \mu(z)^{2}-\varepsilon_{1} \quad(\text { by } 4.12(\mathrm{~F})) \\
& \geq\left(\varepsilon_{0} t(z) \kappa(z)\right)^{4} \cdot t(z)^{-3}-2 \varepsilon_{1}=\left(\varepsilon_{0} \kappa(z)\right)^{4} t(z)-2 \varepsilon_{1}
\end{aligned}
$$

(when we look at the sizes of $\alpha, t(z)$ in 4.3, 4.7 and apply 4.12(E))

$$
\geq \alpha^{-1 / 2}\left|h_{1}(z)\right|^{2} \cdot \kappa(z)^{2}-\varepsilon^{4} \text {. }
$$

4.23 From 4.21 and 4.22 we now get that under our assumptions:

$$
\begin{aligned}
& \rho(f(z)+h(z))-\rho(f(z)) \geq-\left|\mathbf{P}^{2}\left(h_{1}(z)\right)\right|+\alpha^{-1 / 2}\left|h_{1}(z)\right|^{2} \cdot \kappa(z)^{2}-3 \varepsilon^{4} \\
& \quad \geq-C\left|h_{1}(z)\right|^{2}+\alpha^{-1 / 2}\left|h_{1}(z)\right|^{2} \cdot \kappa(z)^{2}-3 \varepsilon^{4} \quad \text { (in view of 4.3) } \\
& \quad \geq\left|h_{1}(z)\right|^{2}\left(\alpha^{-1 / 2} \kappa(z)^{2}-\alpha^{-1 / 4}\right)-3 \varepsilon^{4} .
\end{aligned}
$$

Proposition 4.15 is now proved.

4.24 A globalization process as in [D1, 1.34-1.38] and in 3.15 above will now be applied. There exists then a $C^{\infty}$ map $g_{1}: \bar{D} \rightarrow \mathbb{C}^{M}$ which is holomorphic in $D$ such that:

(i) for all $z \in W^{\prime}:\left|g_{1}(z)-h(z) / S(z)\right|<\left(\varepsilon_{1}\right)^{1 / 2}$,

(ii) for all $z \in \bar{D} \backslash W^{\prime}:\left|g_{1}(z)\right|<\left(\varepsilon_{1}\right)^{1 / 2}$.

Define for $z \in \bar{D}: g(z)=g_{1}(z) \cdot S(z)$. As in Lemma 1, we can take $g=h$ in the case $D=B^{N}$, and skip the globalization process.

4.25 Take $z \in \bar{D}$. To prove (a) of Lemma 2 look separately at the case where $f(z) \in \Omega \backslash G^{\prime}$ and the case where $f(z) \in G^{\prime}$. In the first case $|g(z)|<\left(\varepsilon_{1}\right)^{1 / 3}<$ (by $4.3,4.3)<d\left(f(z), b \Omega\right.$ ). In the second case $|g(z)|<\delta_{0}$ (by $4.14(\mathrm{G})$ ) and therefore (by 4.1, 4.4) $|g(z)|<d(f(z), b \Omega)$. Therefore in both cases $f(z)+g(z) \in \Omega$.

4.26 To prove (b) and (c) look first at the case where either $z \in \bar{D} \backslash W^{\prime}$ or $f(z) \in \Omega \backslash G^{\prime}$. In this case $|g(z)|<\left(\varepsilon_{1}\right)^{1 / 3}$ and by 4.4 we get that

$$
|\rho(f(z)+g(z))-\rho(f(z))|<\varepsilon^{10} .
$$

Thus both (b) and (c) hold in this case. Assume now that $z \in W^{\prime}, f(z) \in G^{\prime}$, then by $4.25 f(z)+g(z) \in G^{\prime \prime}$ and therefore by 4.24 and 4.4: $\mid \rho(f(z)+g(z))$ $-\rho(f(z)+h(z)) \mid<\varepsilon^{10}$. From this, 4.15, and 4.12(E) follows (c). We also get from 4.15 that: $\rho(f(z)+g(z))-\rho(f(z))>\left|h_{1}(z)\right|^{2}-2 \varepsilon^{2}$ and when we look at 4.12(F), (E), 4.14(B) and 4.3 we get: $\rho(f(z)+g(z))-\rho(f(z))>\alpha|g(z)|^{4}-3 \varepsilon^{2}$, and (b) of Lemma 2 follows.

4.27 Now when $z \in U \cap f^{-1}(G)$ then by $4.12(\mathrm{~F}), 4.14(\mathrm{E})$ and $4.7|g(z)|>$ $2 \varepsilon_{0} \alpha-\left(\varepsilon_{1}\right)^{1 / 3}$ and (d) follows. Finally (e) follows from 4.12(A), 4.14(A) and 4.3-4.5; (f) from 4.24 (or from 3.3 when $D=B^{N}$ ). 
Proof of Theorem 3. Fix, until the end of the proof, $D \subset \subset \mathbb{C}^{N}(N \geq 2)$, a $C^{\infty}$-smooth strongly pseudoconvex domain. By the comments before and after Lemma 10, all parts of the theorem will be proved if we show that:

(i) Lemma 1 holds with the assumption that $\rho$ is $(N+1)$-dimensionalplurisubharmonic;

(ii) Lemma 2 holds with the assumption that $\rho$ is $M_{0}$-dimensional-plurisubharmonic, where $M_{0}=\max \{2 N, 5\}$;

(iii) Lemma 2 holds for a critical point which is $(N+1)$-dimensional-convex point of $\rho$.

To show this we need to insert a few additional components to the proof of Lemmas 1, 2 above. Let us start with the first part, the proof of (i). For a ball $B \subset \mathbb{C}^{M}$ we will denote by $B^{\prime}$ the ball with the same center and twice the radius. We go back to the statement of Lemma 1 and recall the assumption that $\rho$ has no critical points in $K_{1}$. Take $w \in K_{1}$; then there exists $V$, an $(N+1)$-dimensional linear complex subspace of $\mathbb{C}^{M}$, such that $\rho_{w, V}$, the restriction of $\rho$ to $w+V$, is strictly plurisubharmonic in a small neighborhood of $w$. Now if we take a small enough ball $B \subset \mathbb{C}^{M}$ centered in $w$ we can assume that for some constant $c>0$, and for every $v \in B: \rho_{v, V}$ is a strictly plurisubharmonic normalized- $c$ function in $V \cap B$. The compactness of $K_{1}$ implies that we can find balls $B_{1}, \ldots, B_{n}$ that cover $K_{1}$, and $V_{1}, \ldots, V_{n}$, $(N+1)$-dimensional linear complex subspaces of $\mathbb{C}^{M}$, and $c>0$, such that for all $1 \leq i \leq n$ and for all $w \in B_{i}^{\prime}: \rho_{w, V_{i}}$ is a strictly plurisubharmonic normalized- $c$ function $V_{i} \cap B_{i}^{\prime}$. It is clear that Lemma 1 is proved for $K_{1}$ if it is proved for $K_{1} \cap \bar{B}_{i}$, for every $1 \leq i \leq n$. Take $1 \leq i \leq n$. It will be proved for $K_{1} \cap \bar{B}_{i}$ with the following (sufficient) estimate in (b): $\rho((f+g)(z))-\rho(f(z))>$ $(c / 2)|g(z)|^{2}-\varepsilon$. The rest of the statement of the lemma is unchanged. To do so one modification is needed in the proof of Lemma 1: in 3.6 we add the assumption that $\left\{u_{1}(z), \ldots, u_{N}(z)\right\} \subset V_{i}$ for all $z \in b D$. Lemmas 4-5 allow us to do so. The rest of the proof is left unchanged with the exception of minor adjustments of the epsilons (in the error terms) and of constants relating to the normalization factor.

Proof of (ii). Here we get into the proof of Lemma 2 and assume in 4.1 that $\delta>0$ was chosen small enough so that for an appropriate $M_{0}$-dimensional complex linear subspace $V$ and $c>0$ (both depend only on $w_{0}$ ) and all $w \in$ $B=\bar{B}\left(w_{0}, 4 \delta\right): \rho_{w, V}$ is a strictly plurisubharmonic normalized- $c$ function in $V \cap B$. We now go through all the stages of the proof of Lemma 2 with one significant change: we assume in 4.8 (as we may, by Lemmas 4-7) that $u, v_{1}, \ldots, v_{N}: b D \rightarrow b B^{M} \cap V$. The rest of the proof stays the same, except for a few constants that must be adjusted in accordance to the normalization constant.

Proof of (iii). Here we need a relatively small part of the proof of Lemma 2 (it can also be done with the proof of Lemma 1). Let $w_{0} \in \Omega$ be a critical point of $\rho$ which is an $(N+1)$-dimensional-convex point of $\rho$. Let $V \subset \mathbb{C}^{M}$ be an $(N+1)$-dimensional subspace such that $\rho_{w_{0}, V}$ is a strictly convex function (see Definition 1.5) in $w_{0}$. Take (in 4.1) $\delta>0$, small enough so that for all $w \in B=\bar{B}\left(w_{0}, 4 \delta\right): \rho_{w, V}$ is a strictly plurisubharmonic normalized- $c$ function in $V \cap B$ (for some constant $c>0$ ). We can ignore any part of the proof of Lemma 2 that relates to the form $P($,$) . Then in 4.8$ omit the 
function $u$ and have $v_{1}, \ldots, v_{N}: b D \rightarrow b B^{M} \cap V$. We can do so by Lemmas 4-5. The map $h_{1}$ is defined as there and the map $h_{2}$ is not needed. Finally we will have $h=h_{1}$. The easier parts of the proof of Lemma 2 would suffice to show that the resulting $g$ fulfils all the requirements of Lemma 2 .

\section{ACKNOWLEDGMENT}

This paper was presented at the 3rd Analysis Colloquium, Berne, summer 1994. I would like to thank the Edmund Landau Center for research in Mathematical Analysis and the Minerva Foundation (Germany) for their support of my participation. I would also like to thank the organizers $\mathrm{K}$. Diedrich and H. M. Reimann for giving me the opportunity for this presentation. The main results of this paper were also presented in lectures given at the University of Michigan, Ann Arbor, and the University of Chicago. I would also like to thank J. E. Fornaess, and R. Narasimhan for their kind invitations.

\section{REFERENCES}

[D1] A. Dor, Proper holomorphic maps between balls in one co-dimension, Ark. Mat. 28 (1990), 49-100.

[D2] Continuous proper holomorphic maps into bounded domains, Proc. Amer. Math. Soc. 119 (1993), 1145-1155.

[D3] - Approximation by proper holomorphic maps into convex domains, Ann. Scuola Norm. Sup. Pisa Ser. (4) 20 (1993), Fasc. 1.

[D4] _ Lifting of proper holomorphic maps, preprint.

[D5] _ A domain in $\mathbf{C}^{m}$ that contains no proper images of the unit disc, Math. Z. (to appear).

[EG] Y. Eliashberg and Mikhael Gromov, Embeddings of Stein manifolds of dimension $n$ into the affine space of dimension 3n/2 + 1, Ann. of Math. 136 (1992), 123-135.

[FG] F. Forstnerič and J. Globevnik, Discs in pseudoconvex domains, Comment. Math. Helv. 67 (1992), 129-145.

[G] J. Globevnik, Relative embeddings of discs into convex domains, Invent. Math. 98 (1989), 331-350.

[H] M. Hakim, Applications propres continues de domaines strictement pseudoconvexes de $\mathbf{C}^{n}$ dans la boule unite $\mathbf{C}^{n+1}$, Duke Math. J. 60 (1990), 115-133.

[K] S. Krantz, Function theory of several complex variables, Wiley, New York, 1982.

[N] A. Noel and B. Stensones, Proper holomorphic maps from weakly pseudoconvex domains, preprint.

[Ra] M. Range, Holomorphic functions and integral representations in several complex variables, Springer-Verlag, New York, 1986.

[Ru] W. Rudin, Function theory in the unit ball of $\mathbf{C}^{n}$, Springer, New York, 1980.

[S] S. Stensones, Proper holomorphic mappings from strongly pseudoconvex domains in $\mathbf{C}^{2}$ to the unit polydisc in $\mathbf{C}^{3}$, Math. Scand. 65 (1989), 129-139.

Institute of Mathematics, Hewbrew University, Givat Ram, Jerusalem, 91904 IsRael Current address: Negev College, D.N. Hof Ashkelon 79165, Israel

E-mail address: csweinba@weizmann.weizmann.ac.il 